\title{
The influence of model parameters on the prediction of gravitational wave signals from stellar core collapse
}

\author{
S. Scheidegger, R. Käppeli, S. C. Whitehouse, T. Fischer, and M. Liebendörfer
}

Department of Physics, University of Basel, Klingelbergstrasse 82, 4056 Basel, Switzerland

e-mail: simon.scheidegger@unibas.ch

Received 31 August 2009 / Accepted 18 January 2010

\begin{abstract}
We present a gravitational wave $(\mathrm{GW})$ analysis of an extensive series of three-dimensional magnetohydrodynamical core-collapse simulations. Our 25 models are based on a $15 M_{\odot}$ progenitor stemming from (i) stellar evolution calculations; (ii) a spherically symmetric effective general relativistic potential, either the Lattimer-Swesty (with three possible compressibilities) or the Shen equation of state for hot, dense matter; and (iii) a neutrino parametrisation scheme that is accurate until about $5 \mathrm{~ms}$ postbounce. For three representative models, we also included long-term neutrino physics by means of a leakage scheme, which is based on partial implementation of the isotropic diffusion source approximation (IDSA). We systematically investigated the effects of the equation of state, the initial rotation rate, and both the toroidal and the poloidal magnetic fields on the GW signature. We stress the importance of including of postbounce neutrino physics, since it quantitatively alters the GW signature. Slowly rotating models, or those that do not rotate at all, show GW emission caused by prompt and proto-neutron star (PNS) convection. Moreover, the signal stemming from prompt convection allows for the distinction between the two different nuclear equations of state indirectly by different properties of the fluid instabilities. For simulations with moderate or even fast rotation rates, we only find the axisymmetric type I wave signature at core bounce. In line with recent results, we could confirm that the maximum GW amplitude scales roughly linearly with the ratio of rotational to gravitational energy at core bounce below a threshold value of about $10 \%$. We point out that models set up with an initial central angular velocity of $2 \pi \mathrm{rad} \mathrm{s}^{-1}$ or faster show nonaxisymmetric narrow-band GW radiation during the postbounce phase. This emission process is caused by a low $T /|W|$ dynamical instability. Apart from these two points, we show that it is generally very difficult to discern the effects of the individual features of the input physics in a GW signal from a rotating core-collapse supernova that can be attributed unambiguously to a specific model. Weak magnetic fields do not notably influence the dynamical evolution of the core and thus the GW emission. However, for strong initial poloidal magnetic fields $\left(\gtrsim 10^{12} \mathrm{G}\right)$, the combined action of flux-freezing and field winding leads to conditions where the ratio of magnetic field pressure to matter pressure reaches about unity which leads to the onset of a jet-like supernova explosion. The collimated bipolar out-stream of matter is then reflected in the emission of a type IV GW signal. In contradiction to axisymmetric simulations, we find evidence that nonaxisymmetric fluid modes can counteract or even suppress jet formation for models with strong initial toroidal magnetic fields. The results of models with continued neutrino emission show that including of the deleptonisation during the postbounce phase is an indispensable issue for the quantitative prediction of GWs from core-collapse supernovae, because it can alter the GW amplitude up to a factor of 10 compared to a pure hydrodynamical treatment. Our collapse simulations indicate that corresponding events in our Galaxy would be detectable either by LIGO, if the source is rotating, or at least by the advanced LIGO detector, if it is not or only slowly rotating.
\end{abstract}

Key words. gravitational waves - supernovae: general - hydrodynamics - neutrinos - stars: rotation - stars: neutron

\section{Introduction}

Gravitational wave (GW) astronomy may soon become a reality and will allow humankind to address questions about many different astrophysical objects that are hidden from the electromagnetic detection. Within the past few years, the first generation of the ground-based GW detectors LIGO (USA), VIRGO (Italy), GEO600 (Germany), and TAMA (Japan) have got very close to or even reached design sensitivity and collected partially coincident data, as discussed by Abbott et al. (2009). Lately, the two $4 \mathrm{~km}$ LIGO detectors were upgraded to sensitivities increased by a factor of 2-3 (enhanced LIGO, see Adhikari et al. 2006) and resumed observations in 2009. Upgrades of the three LIGO interferometers and VIRGO are expected to be completed by 2014 and will increase the observable volume by a factor of $\sim 1000$. Operating at such a high level of precision, GW detectors are sensitive to many different sources, such as compact binary coalescence from black-holes $(\mathrm{BH})$ and neutron stars up to distances of several 100 megaparsecs, but might also very likely produce the first detections of black-hole and neutron star mergers, corecollapse supernovae, and neutron star normal mode oscillations, as recently reviewed in Sathyaprakash \& Schutz (2009).

The broad majority of GW sources can be subdivided into two classes: (i) mathematically well-posed events such as $\mathrm{BH}-$ BH coalescence; and (ii) scenarios that involve matter. The first category of sources can be modelled accurately, the waveform can be calculated with high precision, and thus a matched-filter analysis can be applied. On the other hand, the latter kind of GW sources can only be modelled imperfectly, as matter effects carry large physics uncertainties and open up a huge parameter space for initial conditions. Moreover, even if there were "perfect" models, there is still a turbulent, stochastic element in some GW emission mechanisms that makes it strictly impossible to compute templates. As a result, more general, un-modelled burst analysis techniques are used, as summarised in Abbott et al. (2009) and references therein. One of the scenarios where burst 
data analysis must be applied is the stellar core collapse, where in addition to the matter effects even the fundamental explosion mechanism is not fully settled. It is still unclear which processes may convert some of the released gravitational binding energy of order $O\left(10^{53}\right)$ erg into the typically observed kinetic and internal energy of the ejecta of $O\left(10^{51}\right)$ erg $=1$ Bethe [B] needed to blow off the stellar envelope. While most state-of-the art simulations investigate a mechanism based on neutrino heating in combination with hydrodynamical instabilities, such as Marek \& Janka (2009), others explore the alternative magneto-rotational mechanism (Leblanc \& Wilson 1970; Kotake et al. 2004a; Burrows et al. 2007a; Mikami et al. 2008; Takiwaki et al. 2009), or the acoustic mechanism which is driven by strong core g-modes (Burrows et al. 2006, 2007b). For a recent review see Janka et al. (2007). Takahara \& Sato (1988), Gentile et al. (1993), and lately also Nakazato et al. (2008) and Sagert et al. (2009) reported that a QCD phase transition may power a secondary shock wave which triggers a successful hydrodynamical explosion.

Beside neutrinos, which have already been observed in the context of stellar core collapse of SN1987A (Hirata et al. 1988), GWs could provide access to the electromagnetically hidden compact inner core of some such cataclysmic events. Since a core-collapse supernova is expected to show aspherical features (Leonard et al. 2006), there is reasonable hope that a tiny amount of the released binding energy will be emitted as GWs which could then provide us with valuable information about the angular momentum distribution (Dimmelmeier et al. 2008) and the baryonic equation of state (EoS) (Marek et al. 2009), both of which are uncertain. Furthermore, they might help to constrain theoretically predicted SN mechanisms (Ott 2009). Given the actual sensitivities of today's operating ground-based GW observatories (Whitcomb 2008) combined with knowledge of waveforms from state-of-the art modelling, GWs from a Galactic core-collapse supernova may be considered to be within the detector limits. The collapsing iron core and the subsequently newly formed PNS can be subject to a whole variety of asymmetric hydrodynamical and nonaxisymmetric instabilities which give rise to $\mathrm{GW}$ emission.

In this context probably most attention during the past three decades has been paid to rotational core collapse and bounce dynamics. In this particular phase, the core spins up due to angular momentum conservation, resulting in an oblate and time-dependent deformation that leads to strong GW emission (Müller 1982; Mönchmeyer et al. 1991; Janka \& Müller 1996; Zwerger \& Müller 1997; Rampp et al. 1998; Kotake et al. 2003, 2004b; Obergaulinger et al. 2006; Ott et al. 2004, 2007a,b; Dimmelmeier et al. 2002, 2007, 2008; Scheidegger et al. 2008). The steady improvement of the models (e.g. the inclusion of GR, a micro-physical EoS, treatment of neutrino physics) recently led to a theoretically well understood single and generic, socalled type I wave form which is characterised by a large negative peak at core bounce, followed by ring-down oscillations that damp quickly (Ott et al. 2007a,b; Dimmelmeier et al. 2007, 2008; with detailed references therein). Despite the reduction to a single wave form, the combined information of the GW amplitude and the location of the narrow peak of the GW spectral energy density in frequency space contains information that makes it still possible to constrain progenitor- and postbounce rotation, but can barely distinguish between different finite-temperature EoS (see e.g. Dimmelmeier et al. 2008, who used the EoS of Lattimer \& Swesty 1991 and Shen et al. 1998b).

Gravitational waves from magneto-rotational collapse were considered in detail by Kotake et al. (2004b); Shibata et al. (2006) and Obergaulinger et al. (2006). As main differences compared to simulations which do not include magnetic fields it was found by these groups that only in the very special case of precollapse fields as strong as $\gtrsim 10^{12} \mathrm{G}$ the overall dynamics can be influenced. As Heger et al. (2005) have argued, such strong fields are unlikely to occur in standard core-collapse supernova progenitors. The GW amplitude is then affected by (i) time-dependent magnetic fields, which contribute considerably to the overall energy density; and (ii) by bipolar magnetohydrodynamic (MHD) jet outflows which give rise to a socalled type IV signal with memory (Obergaulinger et al. 2006). Physically, such a memory effect in the GW signal arises from the temporal history of asymmetric matter outflow, leaving behind a constant offset in the amplitude (Thorne 1989).

Recently it has been argued through numerical simulations of equilibrium neutron star models or full core-collapse simulations that PNSs with a high degree of differential rotation can be subject to nonaxiymmetric rotational instabilities at low $\beta$ values $(=T /|W|$, ratio of rotational to gravitational energy), leading to strong narrow-band GW emission (Saijo et al. 2003; Watts et al. 2005; Ott et al. 2005; Saijo \& Yoshida 2006; Ou \& Tohline 2006; Ott et al. 2007b; Cerdá-Durán et al. 2007; Ott et al. 2007a; Scheidegger et al. 2008). However little is known about the true nature of the instability at present. Previous work has failed to establish an analytical instability criterion, and the dependence of the instability on PNS rotation rate and degree of differential rotation is still unclear, as it was pointed out by Ott (2009).

Current state-of-the-art stellar evolution calculations (Heger et al. 2005) tell us that iron cores of stars generally lose most of their angular momentum during their evolution due to magnetic torques. Therefore, they cannot become subject to strong rotationally-induced aspherities. However, anisotropic neutrino emission, convection and standing accretion shock instability (SASI, see e.g. Blondin et al. 2003) - driven deviations from spherical symmetry which can lead to the emission of GWs of sizable amplitudes are likely to occur inside the PNS and the post-shock gain region and last for probably hundreds of ms (Müller \& Janka 1997; Müller et al. 2004; Kotake et al. 2007; Marek et al. 2009; Murphy et al. 2009; Kotake et al. 2009a,b). Although there is qualitative consensus among the different core-collapse supernova groups about the aforementioned features being emitters of stochastic broad-band signals, their detailed quantitative character still remains quite uncertain, since self-consistent 3D simulations with proper long-term neutrino transport were not carried out so far, but would in principle be required. The most recent, very elaborate $2 \mathrm{D}$ simulations in that context were performed by Marek et al. (2009). Their numerical setup includes long-term multi-flavor neutrino transport, an effective relativistic potential and two different EoS. The first $100 \mathrm{~ms}$ of after bounce they observed GWs from early prompt postbounce convection, peaking somewhat around or below $100 \mathrm{~Hz}$, depending on the employed EoS. After this early episode, the GW emission in their models is dominated by a growing negative amplitude related to anisotropic neutrino emission at frequencies $\lesssim 200 \mathrm{~Hz}$, while the GW signal associated with non-radial mass motions stems from density regimes $10^{11}-10^{13} \mathrm{~g} \mathrm{~cm}^{-3}$ and peaks in the frequency range of $300-800 \mathrm{~Hz}$, highly sensitive to the nuclear EoS.

In the core-collapse scenario, PNS pulsations can provide another mechanism for GW emission. Ott et al. (2006a) pointed out that in the context of the acoustic mechanism (Burrows et al. 2006) excited core g-mode oscillations might emit very strong GWs. The GW signal is due to the nonlinear quadrupole components of the pulsations that, at least initially, are of $l=1 \mathrm{~g}$-mode character (Ott et al. 2006a). 
For recent reviews on GWs from core-collapse supernovae with complete lists of references see Ott (2009) and Kotake et al. (2006).

In this paper, we present the gravitational wave analysis of a comprehensive set of three-dimensional MHD core-collapse supernova simulations in order to investigate the dependencies of the resulting GW signal on the progenitor's initial conditions. Our calculations encompass presupernova models from stellar evolution calculations, a finite-temperature nuclear EoS and a computationally efficient treatment of the deleptonisation and neutrino emission during the collapse. General relativistic corrections to the spherically symmetric Newtonian gravitational potential are taken into account. Moreover, several models incorporate long-term neutrino physics by means of a leakage scheme. As for the progenitor configuration, we systematically consider not only variations in the precollapse rotation rate, but also in the nuclear EoS and the magnetic field topology. This study extends the work of Scheidegger et al. (2008), who investigated only two models with similar input physics. In this way, we carried out the so far largest parameter study of 3D MHD stellar collapse with respect to the prediction of GWs.

The structure of the paper is as follows. In Sect. 2 we briefly describe the numerical methods, input physics and progenitor configurations applied in our core-collapse simulations. Furthermore, we describe how we extract GWs from our model set. In Sect. 3 we discuss results of 25 three-dimensional MHD simulations with respect to the GW signal from convection, rotational core bounce, nonaxisymmetric rotational instabilities and very strong magnetic fields. In Sect. 4, we summarise and present conclusions.

\section{Numerical methods}

\subsection{The $3 D M H D$ code and its input physics}

The algorithm used to solve the time-dependent, Newtonian MHD equations in our 3D simulations is based on a simple and fast cosmological MHD code of Pen et al. (2003); Liebendörfer et al. (2006), which has been parallelised with a hybrid combination of MPI and openMP and improved and adapted to the requirements of core-collapse supernova simulations (Käppeli et al. 2009). The 3D computational domain consists of a central cube of $600^{3} \mathrm{~km}^{3}$ volume, treated in equidistant Cartesian coordinates with a grid spacing of $1 \mathrm{~km}$. It is, as explained in detail in Scheidegger et al. (2008), embedded in a larger spherically symmetric computational domain that is treated by the time-implicit hydrodynamics code "Agile" (Liebendörfer et al. 2002). With this setup, the code scales nicely to at least 8000 parallel processes (Käppeli et al. 2009). The ideal MHD equations read

$$
\begin{aligned}
& \frac{\partial \rho}{\partial t}+\nabla \cdot(\rho \boldsymbol{v})=0 \\
& \frac{\partial \rho \boldsymbol{v}}{\partial t} \nabla \cdot(\boldsymbol{v} \rho \boldsymbol{v}-\boldsymbol{b} \boldsymbol{b})+\nabla P=-\rho \nabla \Phi \\
& \frac{\partial E}{\partial t}+\nabla \cdot[(E+P) \boldsymbol{v}-\boldsymbol{b} \cdot(\boldsymbol{v} \cdot \boldsymbol{b})]=-\rho \boldsymbol{v} \cdot \nabla \Phi \\
& \frac{\partial \boldsymbol{b}}{\partial t}-\nabla \times(\boldsymbol{v} \times \boldsymbol{b})=0
\end{aligned}
$$

expressing the conservation of mass, momentum, energy and magnetic flux, respectively. $\rho$ stands for mass density, $\mathbf{v}$ is the velocity vector and $E=\rho e+\frac{\rho}{2} v^{2}+\frac{b^{2}}{2}$ the total energy (the sum of internal, kinetic and magnetic energy). The magnetic field is given by $\boldsymbol{B}=\sqrt{4 \pi} \boldsymbol{b}$ and the total pressure by $P=p+\frac{b^{2}}{2}$ (the sum of gas and magnetic pressure). The MHD equations are evolved from a condition

$\nabla \cdot \boldsymbol{b}=0$

We employ the constrained transport method (Evans \& Hawley 1988) to guarantee the divergence-free time evolution of the magnetic field. The right hand side of Eqs. (2) and (3) take into account the effect of gravitational forces on the magnetohydrodynamical variables. The gravitational potential $\Phi$ obeys the Poisson equation

$\Delta \Phi=4 \pi G \rho$.

We only implement the monopole term of the gravitational potential by a spherically symmetric mass integration that includes general relativistic corrections (Marek et al. 2006). In this approach, the Newtonian gravitational potential $\Phi$ is replaced by an effective potential $\Phi_{\text {eff }}$ ("case A" potential, see Marek et al. 2006)

$\Phi_{\mathrm{eff}}(r)=\int_{r}^{\infty} \frac{\mathrm{d} r^{\prime}}{r^{\prime 2}}\left[\frac{m_{\mathrm{eff}}}{4 \pi}+r^{\prime 3} \cdot\left(p+p_{\nu}\right)\right] \cdot \frac{1}{\Gamma^{2}}\left(\frac{\rho+e+p}{\rho}\right)$

with $p$ being the gas pressure, $p_{v}$ the neutrino pressure and $e$ the internal energy density. The effective mass is given by

$m_{\mathrm{eff}}(r)=4 \pi \int_{0}^{r} \mathrm{~d} r^{\prime} r^{\prime 2}(\rho+e+E)$,

where $E$ is the neutrino energy. The metric function $\Gamma$ is given by

$\Gamma=\sqrt{1+v_{r}^{2}-\frac{2 m_{\mathrm{eff}}}{r}}$,

where $v_{\mathrm{r}}$ is the radial fluid velocity. Note that Müller et al. (2008) recently proposed an effective relativistic gravitational potential for rapidly rotating configurations. However, since it would have added another degree of freedom to our parameter set, we did not consider it in this study.

The system of the MHD equations must be closed by a finite-temperature EoS. In core-collapse supernova simulations, an EoS has to handle several different regimes. For temperatures below $0.5 \mathrm{MeV}$, the presence of nuclei and time dependent nuclear processes dominate the internal energy evolution. For simplicity, an ideal gas of Si-nuclei is assumed, which, for our GW study, is sufficiently accurate to describe the low baryonic pressure contribution at low densities in the outer core. At higher temperatures, where matter is in nuclear statistical equilibrium, we employ two alternative EoS: The Lattimer \& Swesty EoS (Lattimer \& Swesty 1991, LS EoS) and the one by Shen et al. (1998a). The first EoS is based on a phenomenological compressible liquid drop model; it also includes surface effects as well as electron-positron and photon contributions. The LS EoS assumes a nuclear symmetry energy of $29.3 \mathrm{MeV}$ and we will perform simulations with three choices of the nuclear compressibility modulus $\mathrm{K}(180,220,375 \mathrm{MeV})$ as provided by Lattimer \& Swesty (1991). Since variations in K affect the stiffness of the nuclear component of the EoS, this enables us to probe the effects of variations in stiffness while keeping the general EoS model fixed. The pure baryon EoS from Shen et al. (1998a) has a compressibility of $K=281 \mathrm{MeV}$ and a symmetry energy of $36.9 \mathrm{MeV}$. It is based on relativistic mean field theory and the Thomas-Fermi approximation. For matter in non-NSE $(T(<0.44) \mathrm{MeV})$, the Shen EoS is coupled to the baryonic and electron-positron EoS given in Timmes \& Arnett (1999) and Timmes \& Swesty (2000). It employs an ideal gas for the nuclei and additionally includes contributions from ionion-correlations and photons. 


\subsection{The treatment of neutrino physics}

The treatment of neutrino physics is an essential ingredient of core-collapse supernova simulations (Mezzacappa 2005). Multidimensional core-collapse supernova simulations therefore must rely on more or less severe approximations of the neutrino physics. In state-of-the-art 2D simulations, one way in which Boltzmann transport is approximated is the so-called ray-by-ray plus scheme" (Buras et al. 2006b). It solves the full transport in separate $1 \mathrm{D}$ angular segments, where the neighbouring rays are coupled. Other groups rely on multi-group flux limited diffusion (MGFLD, Ott et al. 2008; Swesty \& Myra 2009). MGFLD treats all neutrinos in seperate energy groups, drops the momentum space angular dependence of the radiation field and evolves the zeroth moment of the specific intensity instead of the specific intensity itself. Ott et al. (2008) also compared MGFLD with angle-dependent (i.e. partly Boltzmann) transport in 2D. In three dimensions, simulations have been performed using "grey" fluxlimited diffusion (Fryer \& Warren 2004), which oversimplyfies the important neutrino spectrum. It is important to resolve the neutrino spectrum, since the charge-current interaction rates go with the square of the neutrino energy.

In our 3D MHD simulations, we apply a parametrised deleptonisation scheme (Liebendörfer 2005). Detailed sphericallysymmetric collapse calculations with Boltzmann neutrino transport show that the electron fraction $Y_{\mathrm{e}}$ in different layers in the homologously collapsing core follow a similar deleptonisation trajectory with density. The local electron fraction of a fluid element can be parametrised as a function of density $Y_{\mathrm{e}}(\rho)$. For our 3D simulations, we apply tabulated $Y_{\mathrm{e}}$ profiles which were obtained from detailed general relativistic, spherically symmetric three-flavour Boltzmann neutrino transport. Exemplary profiles are shown in Fig. 1. The offset between the $Y_{\mathrm{e}}$ profiles from the LS- and the Shen EoS is consistent with the different asymmetry energies of the two EoS. This in turn is reflected in different neutrino reaction rates and thus a different $Y_{\mathrm{e}}$ at a given density. These effects have been discussed in Sumiyoshi et al. (2008) and Fischer et al. (2009) for massive progenitor stars in the range of 40-50 $M_{\odot}$. However, this parametrisation scheme is only valid until a few milliseconds after bounce, since it cannot account for the neutronisation burst, as explained in Liebendörfer (2005) and Scheidegger et al. (2008).

In order to give an estimate of how long the above approximation is able to predict quantitatively reliable postbounce (pb) GW signals for times $t \gtrsim 5 \mathrm{~ms}$ after bounce, we carried out three representative simulations $\left(\mathrm{R} 1 \mathrm{E} 1 \mathrm{CA} \mathrm{A}_{L}, \mathrm{R} 3 \mathrm{E} 1 \mathrm{AC}_{L}\right.$ and $\mathrm{R} 4 \mathrm{E} 1 \mathrm{FC} C_{L}$, see Table 1) that incorporate a postbounce treatment for neutrino transport and deleptonisation. The scheme we apply is based on a partial implementation of the isotropic diffusion source approximation (IDSA; Liebendörfer et al. 2009). The IDSA splits the distribution function $f$ of the neutrinos into two components, a trapped component $f^{t}$ and a streaming component $f^{s}$, representing neutrinos of a given species and energy which find the local zone opaque or transparent, respectively. The total distribution function is the sum of the two components, $f=f^{t}+f^{s}$. The two components are evolved using separate numerical techniques, coupled by a diffusion source term. The trapped component transports neutrinos by diffusion to adjacent fluid elements, while in this paper the streaming component is discarded $\left(f^{s}=0\right)$, implying that these neutrinos are lost from the simulation immediately. The part of the IDSA for trapped neutrinos is implemented in three dimensions, including both electron neutrinos and electron anti-neutrinos (Whitehouse \& Liebendörfer 2010, in preparation). The use of this partial

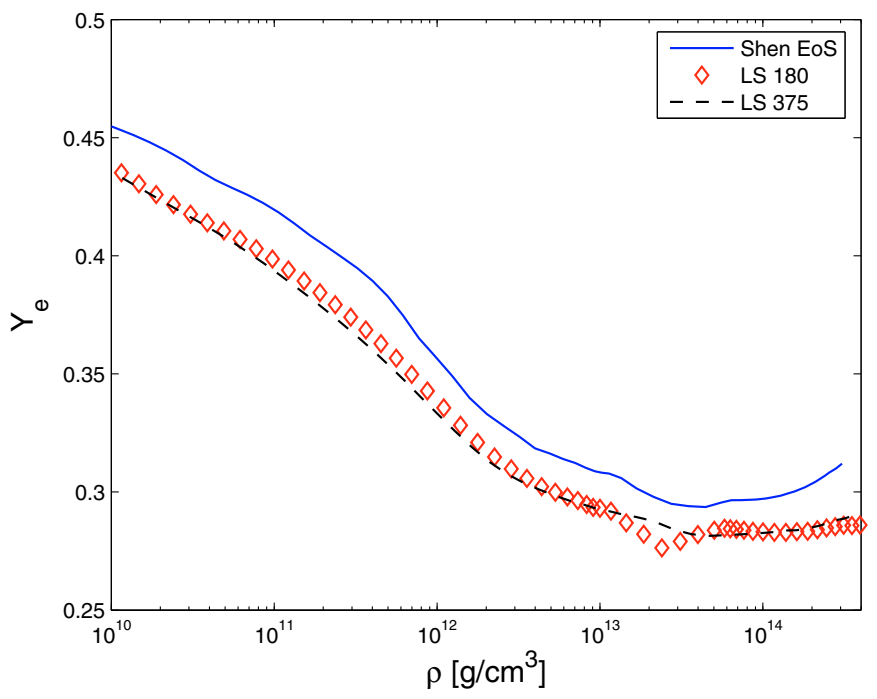

Fig. 1. Electron fraction $Y_{\mathrm{e}}$ as a function of density, obtained from detailed general relativistic, spherically symmetric three-flavour Boltzmann neutrino transport. The calculations were carried out using a $15 M_{\odot}$ progenitor from Woosley \& Weaver (1995) and a finite temperature EoS of either Lattimer \& Swesty (1991) or Shen et al. (1998a). In the figure, we denote the LS EoS version with a compressibility of $180 \mathrm{MeV}$ as LS180, the one carried out with $375 \mathrm{MeV}$ as LS375. The Shen EoS is named as such.

implementation of the IDSA enables us to capture the neutrino burst and to continue our simulations into the postbounce regime, as shown in Fig. 2.

The leakage scheme is switched on at core bounce, when the central density of the core reaches its first maximum value. At present, we neglect the emission of $\mu$ and $\tau$ neutrinos and their antineutrinos, which in principle play an important role in the cooling of the PNS. However, since our leakage schemes neglects any absorption of transported neutrinos, it already overestimates the cooling of the PNS, even without the treatment of the $\mu$ and $\tau$ neutrinos. This is shown in Fig. 3 .

\subsection{Initial model configurations}

We construct the initial conditions of our simulations by a parametric approach. All our models are launched from a $15 M_{\odot}$ progenitor star from the stellar evolutionary calculations of Woosley \& Weaver (1995). Angular momentum was added to the presupernova model according to a shell-type rotation law (Eriguchi \& Müller 1985)

$\Omega(r)=\Omega_{i, c} \cdot \frac{A^{2}}{A^{2}+r^{2}}$,

where we define $r=\sqrt{x^{2}+y^{2}+z^{2}}$ as spherical radius, and $(x, y, z)$ as Cartesian coordinates. The constant $\mathrm{A}$ is the degree of differential rotation that controls the steepness of the angular velocity profile, $\Omega_{i, c}$ the initial central rotation rate, and $r$ is the distance from the origin. In our whole model set we choose $A=500 \mathrm{~km}$ as degree of differential rotation.

In order to guarantee a divergence-free initial state, the initial magnetic field configuration was set up employing its definition via the vector potential $\boldsymbol{A}$. The components of $\boldsymbol{A}$ we chose are

$\boldsymbol{A}=\left(-\frac{B_{\mathrm{pol}}}{2} y, \frac{B_{\mathrm{pol}}}{2} x, \sqrt{x^{2}+y^{2}} B_{\mathrm{tor}}\right)$. 
Table 1. Summary of initial conditions ${ }^{a}$.

\begin{tabular}{|c|c|c|c|c|c|c|c|c|c|}
\hline Model & $\Omega_{c, i}\left[\operatorname{rad~s}^{-1}\right]$ & $\beta_{i}$ & $\beta_{b}$ & EoS & $\rho_{b}\left[\frac{g}{\mathrm{~cm}^{3}}\right]$ & $B_{\mathrm{tor}, i}[\mathrm{G}]$ & $B_{\mathrm{pol}, i}[\mathrm{G}]$ & $E_{m} /|W|_{b}$ & $E_{m} /|W|_{f}$ \\
\hline R0E1CA & 0 & 0 & 0 & E1 & $4.39 \times 10^{14}$ & $5 \times 10^{9}$ & $1 \times 10^{6}$ & $1.8 \times 10^{-9}$ & $3.8 \times 10^{-8}$ \\
\hline R0E3CA & 0 & 0 & 0 & E3 & $4.17 \times 10^{14}$ & $5 \times 10^{9}$ & $1 \times 10^{6}$ & $1.8 \times 10^{-9}$ & $3.1 \times 10^{-8}$ \\
\hline ROSTCA & 0 & 0 & 0 & ST & $3.38 \times 10^{14}$ & $5 \times 10^{9}$ & $1 \times 10^{6}$ & $1.7 \times 10^{-9}$ & $3.3 \times 10^{-8}$ \\
\hline R1E1CA & 0.3 & $0.59 \times 10^{-5}$ & $1.7 \times 10^{-4}$ & E1 & $4.53 \times 10^{14}$ & $5 \times 10^{9}$ & $1 \times 10^{6}$ & $1.7 \times 10^{-9}$ & $3.4 \times 10^{-8}$ \\
\hline R1E3CA & 0.3 & $0.59 \times 10^{-5}$ & $1.7 \times 10^{-4}$ & E3 & $4.17 \times 10^{14}$ & $5 \times 10^{9}$ & $1 \times 10^{6}$ & $1.7 \times 10^{-9}$ & $3.6 \times 10^{-8}$ \\
\hline R1E1DB & 0.3 & $0.59 \times 10^{-5}$ & $1.7 \times 10^{-4}$ & E1 & $4.53 \times 10^{14}$ & $5 \times 10^{10}$ & $1 \times 10^{7}$ & $1.7 \times 10^{-7}$ & $3.0 \times 10^{-6}$ \\
\hline R1STCA & 0.3 & $0.59 \times 10^{-5}$ & $1.7 \times 10^{-4}$ & ST & $3.38 \times 10^{14}$ & $5 \times 10^{9}$ & $1 \times 10^{6}$ & $1.7 \times 10^{-9}$ & $3.2 \times 10^{-8}$ \\
\hline $\mathrm{R} \mathrm{E} 1 \mathrm{CA} \mathrm{A}_{L}$ & 0.3 & $0.59 \times 10^{-5}$ & $1.8 \times 10^{-4}$ & E1 & $4.38 \times 10^{14}$ & $5 \times 10^{9}$ & $1 \times 10^{6}$ & $6.1 \times 10^{-9}$ & $1.2 \times 10^{-7}$ \\
\hline R2E1AC & $\pi$ & $0.64 \times 10^{-3}$ & $1.6 \times 10^{-2}$ & E1 & $4.27 \times 10^{14}$ & $1 \times 10^{6}$ & $1 \times 10^{9}$ & $6.5 \times 10^{-9}$ & $1.27 \times 10^{-5}$ \\
\hline $\mathrm{R} 2 \mathrm{E} 3 \mathrm{AC}$ & $\pi$ & $0.64 \times 10^{-3}$ & $1.6 \times 10^{-2}$ & E3 & $4.00 \times 10^{14}$ & $1 \times 10^{6}$ & $5 \times 10^{9}$ & $6.4 \times 10^{-9}$ & $5.1 \times 10^{-6}$ \\
\hline R2STAC & $\pi$ & $0.64 \times 10^{-3}$ & $1.6 \times 10^{-2}$ & ST & $3.30 \times 10^{14}$ & $1 \times 10^{6}$ & $5 \times 10^{9}$ & $6.4 \times 10^{-9}$ & $3.6 \times 10^{-6}$ \\
\hline R3E1AC & $2 \pi$ & $0.26 \times 10^{-2}$ & $5.2 \times 10^{-2}$ & E1 & $3.80 \times 10^{14}$ & $1 \times 10^{6}$ & $5 \times 10^{9}$ & $8.0 \times 10^{-9}$ & $6.7 \times 10^{-6}$ \\
\hline R3E2AC & $2 \pi$ & $0.26 \times 10^{-2}$ & $5.1 \times 10^{-2}$ & $\mathrm{E} 2$ & $3.65 \times 10^{14}$ & $1 \times 10^{6}$ & $5 \times 10^{9}$ & $8.9 \times 10^{-9}$ & $7.2 \times 10^{-6}$ \\
\hline R3E3AC & $2 \pi$ & $0.26 \times 10^{-2}$ & $5.1 \times 10^{-2}$ & E3 & $3.64 \times 10^{14}$ & $1 \times 10^{6}$ & $5 \times 10^{9}$ & $9.0 \times 10^{-9}$ & $5.8 \times 10^{-6}$ \\
\hline R3STAC & $2 \pi$ & $0.26 \times 10^{-2}$ & $5.1 \times 10^{-2}$ & ST & $3.01 \times 10^{14}$ & $1 \times 10^{6}$ & $5 \times 10^{9}$ & $8.9 \times 10^{-9}$ & $4.7 \times 10^{-6}$ \\
\hline R3E1CA & $2 \pi$ & $0.26 \times 10^{-2}$ & $5.2 \times 10^{-2}$ & E1 & $3.82 \times 10^{14}$ & $5 \times 10^{9}$ & $1 \times 10^{6}$ & $3.1 \times 10^{-9}$ & $1.4 \times 10^{-8}$ \\
\hline R3E1DB & $2 \pi$ & $0.26 \times 10^{-2}$ & $5.2 \times 10^{-2}$ & E1 & $3.81 \times 10^{14}$ & $5 \times 10^{10}$ & $1 \times 10^{7}$ & $3.1 \times 10^{-7}$ & $1.6 \times 10^{-6}$ \\
\hline $\mathrm{R} 3 \mathrm{E} 1 \mathrm{AC}_{L}$ & $2 \pi$ & $0.26 \times 10^{-2}$ & $5.1 \times 10^{-2}$ & E1 & $3.65 \times 10^{14}$ & $1 \times 10^{6}$ & $5 \times 10^{9}$ & $8.7 \times 10^{-9}$ & $8.6 \times 10^{-5}$ \\
\hline R4E1AC & $3 \pi$ & $0.57 \times 10^{-2}$ & $8.6 \times 10^{-2}$ & E1 & $3.22 \times 10^{14}$ & $1 \times 10^{6}$ & $5 \times 10^{9}$ & $1.2 \times 10^{-8}$ & $1.3 \times 10^{-5}$ \\
\hline R4STAC & $3 \pi$ & $0.57 \times 10^{-2}$ & $9.0 \times 10^{-2}$ & ST & $2.69 \times 10^{14}$ & $1 \times 10^{6}$ & $5 \times 10^{9}$ & $1.5 \times 10^{-8}$ & $1.4 \times 10^{-5}$ \\
\hline R4E1EC & $3 \pi$ & $0.57 \times 10^{-2}$ & $8.6 \times 10^{-2}$ & E1 & $3.22 \times 10^{14}$ & $1 \times 10^{11}$ & $5 \times 10^{9}$ & $2.4 \times 10^{-8}$ & $1.3 \times 10^{-5}$ \\
\hline R4E1FC & $3 \pi$ & $0.57 \times 10^{-2}$ & $8.6 \times 10^{-2}$ & E1 & $3.19 \times 10^{14}$ & $1 \times 10^{12}$ & $5 \times 10^{9}$ & $1.3 \times 10^{-4}$ & $1.3 \times 10^{-4}$ \\
\hline $\mathrm{R}_{4} \mathrm{E} 1 \mathrm{FC} C_{L}$ & $3 \pi$ & $0.57 \times 10^{-2}$ & $8.7 \times 10^{-2}$ & E1 & $3.14 \times 10^{14}$ & $1 \times 10^{12}$ & $5 \times 10^{9}$ & $1.3 \times 10^{-4}$ & $2.0 \times 10^{-4}$ \\
\hline $\mathrm{R} 4 \mathrm{E} 1 \mathrm{CF}$ & $3 \pi$ & $0.57 \times 10^{-2}$ & $8.2 \times 10^{-2}$ & E1 & $3.22 \times 10^{14}$ & $5 \times 10^{9}$ & $1 \times 10^{12}$ & $5.8 \times 10^{-4}$ & $5.9 \times 10^{-3}$ \\
\hline R5E1AC & $4 \pi$ & $1.02 \times 10^{-2}$ & $10.2 \times 10^{-2}$ & E1 & $2.47 \times 10^{14}$ & $1 \times 10^{6}$ & $5 \times 10^{9}$ & $1.3 \times 10^{-8}$ & $1.2 \times 10^{-5}$ \\
\hline
\end{tabular}

Notes. ${ }^{(a)}$ The subscript $i$ stands for initial, $b$ for bounce, while $f$ stands for final. $\rho_{b}$ is the maximum central density at the time of core bounce. $B_{\text {pol, } i}$ and $B_{\mathrm{tor}, i}$ abbreviate the initially imposed toroidal and poloidal magnetic fields, whereas $E_{m} /|W|$ stands for the ratio of magnetic to gravitational energy. The subscript $L$ denotes models which were carried out with the leakage scheme.

In order to mimic a dipole-like field, we scale the vector potential with density according to

$\tilde{\boldsymbol{A}}=\sqrt{\frac{\rho}{\rho_{\text {ref }}}} \boldsymbol{A}$.

Finally, the magnetic field is derived from this vector potential

$\boldsymbol{B}=\nabla \times \tilde{\boldsymbol{A}}$.

The initial toroidal- and poloidal components of the magnetic field are specified at a reference density of $\rho_{\text {ref }}=5 \times 10^{7} \mathrm{~g} \mathrm{~cm}^{-3}$ according to Heger et al. (2005).

We compute a total of 25 models, changing the combination of total angular momentum, the EoS, and toroidal- and poloidal magnetic fields. The model parameters are summarised in Table 1. The models are named after the combinations of initial central rotation rate, the EoS, and toroidal- and poloidal magnetic fields. The first two letters of the model name represent the initial central rotation rate $\Omega_{c, i}\left[\mathrm{rad} \mathrm{s}^{-1}\right]$ according to

\begin{tabular}{c|c|c|c|c|c}
0 & 0.3 & $\pi$ & $2 \pi$ & $3 \pi$ & $4 \pi$ \\
\hline R0 & R1 & R2 & R3 & R4 & R5
\end{tabular}

the second two letters stand for the applied EoS

\begin{tabular}{c|c|c|c} 
LS & LS \\
$(K=180 \mathrm{MeV})$ & $(K=220 \mathrm{MeV})$ & $\begin{array}{c}\text { LS } \\
(K=325 \mathrm{MeV})\end{array}$ & Shen \\
\hline E1 & E2 & E3 & ST
\end{tabular}

while the last two letters assign the order of magnitude of the toroidal-, and poloidal field strength [G] according to

\begin{tabular}{c|c|c|c|c|c}
$10^{6}$ & $10^{7}$ & $10^{9}$ & $10^{10}$ & $10^{11}$ & $10^{12}$ \\
\hline $\mathrm{A}$ & $\mathrm{B}$ & $\mathrm{C}$ & $\mathrm{D}$ & $\mathrm{E}$ & $\mathrm{F}$
\end{tabular}.

The final subscript $L$ signs simulations which were carried out with the leakage scheme. Some of the values which we adopt as rotation rate and magnetic fields correspond to the values suggested in Heger et al. (2005). However, we point out that some of the initial rotation rates $\left(\Omega_{c, i} \gtrsim \pi \mathrm{rad} \mathrm{s}^{-1}\right)$ and magnetic fields $\left(B \gtrsim 10^{10} \mathrm{G}\right)$ are larger and stronger compared to current predictions from stellar evolution calculations. However, we computed these models in order to cover a wide parameter space for our three-dimensional models with neutrino transport approximations.

\subsection{Gravitational wave extraction}

The two independent polarisations of the dimensionless gravitational wave field $h_{\mu \nu}=g_{\mu \nu}-\eta_{\mu \nu}$ in the transverse traceless gauge are given by

$h_{i j}^{T T}(\boldsymbol{X}, t)=\frac{1}{R}\left(A_{+} e_{+}+A_{\times} e_{\times}\right)$.

The spatial indices $i, j$ run from 1 to 3 (Misner et al. 1973). $R$ is the distance from the source to the observer and the unit polarisation tensors $e_{+}$and $e_{\times}$in spherical coordinates are represented as

$e_{+}=e_{\theta} \otimes e_{\theta}-e_{\phi} \otimes e_{\phi}$
$e_{\times}=e_{\theta} \otimes e_{\phi}+e_{\phi} \otimes e_{\theta}$.

In the slow-motion limit (Misner et al. 1973; Finn \& Evans 1990) the amplitudes $A_{+}$and $A_{\times}$are linear combinations of the second time derivative of the transverse traceless mass quadrupole 


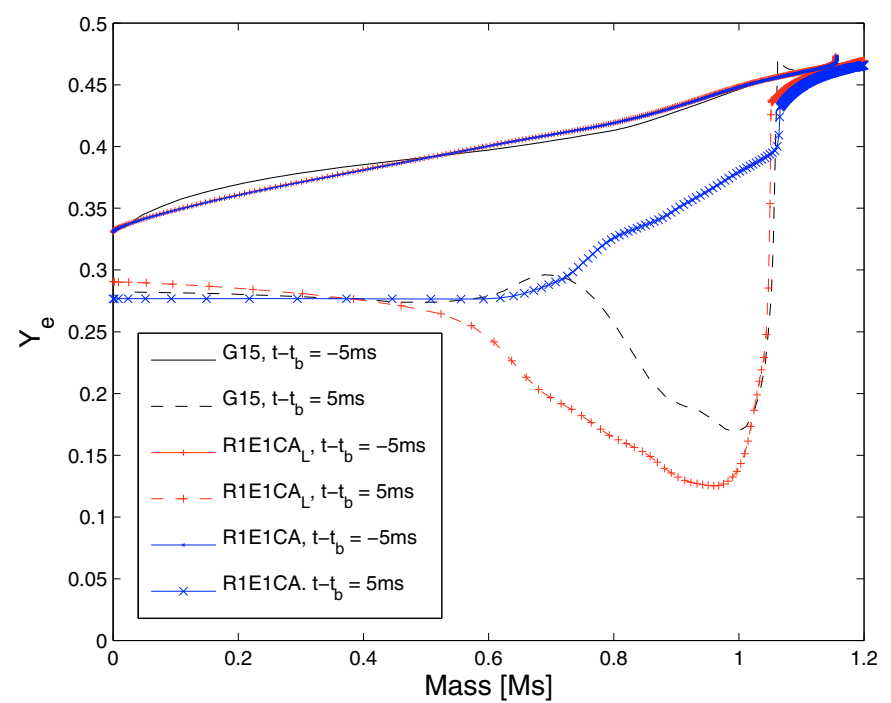

Fig. 2. Comparison of the $Y_{\mathrm{e}}$ profiles of the almost non-rotating 3D models R1E1CA and R1E1CA $A_{L}$ with the spherically symmetric model G15, which is based on general relativistic three-flavour neutrino Boltzmann transport (Liebendörfer et al. 2005) as function of the enclosed mass at $5 \mathrm{~ms}$ before and after bounce. Note that while the simulation R1E1CA, which includes only the neutrino parametrisation scheme for the collapse phase, cannot model the neutrino burst, the "leakage"-IDSA model R1E1CA can.

tensor $t_{i j}^{T T}$. In Cartesian coordinates, the quadrupole tensor is expressed as

$t_{i j}^{T T}=\frac{G}{c^{4}} \int \mathrm{d} V \rho\left[x_{i} x_{j}-\frac{1}{3} \delta_{i j}\left(x_{1}^{2}+x_{2}^{2}+x_{3}^{2}\right)\right]^{T T}$.

For convenience we evaluate below the GW amplitudes along the polar axis $(\theta=\phi=0$, denoted as subscript I)

$A_{+\mathrm{I}}=\ddot{t}_{x x}^{T T}-\ddot{t}_{y y}^{T T}$,

$A_{\times \mathrm{I}}=2 \ddot{t}_{x y}^{T T}$,

and in the equatorial plane $\left(\theta=\frac{\pi}{2}, \phi=0\right.$, denoted as II)

$A_{+\mathrm{II}}=\ddot{t}_{z z}^{T T}-\ddot{t}_{y y}^{T T}$,

$A_{\times \mathrm{II}}=-2 \ddot{t}_{y z}^{T T}$.

Since a direct evaluation of Eq. (17) is numerically problematic, as discussed in Finn \& Evans (1990), we apply alternative reformulations of the standard quadrupole formula, in which one or both time derivatives are replaced by hydrodynamic variables using the continuity and momentum equations. In the first moment of momentum density formula (see Finn \& Evans 1990), the first time derivative of the quadrupole moment yields

$\dot{t}_{i j}^{T T}=\frac{G}{c^{4}} \int \mathrm{d} V \rho\left[v_{i} x_{j}+v_{j} x_{i}-\frac{2}{3} \delta_{i j}\left(v_{1} x_{1}+v_{2} x_{2}+v_{3} x_{3}\right)\right]^{T T}$.

In the stress formulation Blanchet et al. (1990), $\dot{t}_{i j}^{T T}$ is given by

$\ddot{t}_{i j}^{T T}=\frac{G}{c^{4}} \int \mathrm{d} V \rho\left(2 v_{i} v_{j}-x_{i} \partial_{j} \Phi_{\mathrm{eff}}-x_{j} \partial_{i} \Phi_{\mathrm{eff}}\right)^{T T}$,

where $\Phi_{\text {eff }}$ is the gravitational potential. We apply both ways of computing the GW signal and compare them by computing the
Table 2. Overlap.

\begin{tabular}{ccr}
\hline \hline Model & $\Omega_{c, i}\left[\mathrm{rad} \mathrm{s}^{-1}\right]$ & Overlap \\
\hline R1E1AC $_{L}$ & 0.3 & $96 \%$ \\
R3E1AC $_{L}$ & $2 \pi$ & $92 \%$ \\
R4E1AC $_{L}$ & $3 \pi$ & $97 \%$ \\
\hline
\end{tabular}

overlap (Giacomazzo et al. 2009) of the resulting waveforms via

$$
\begin{aligned}
& O\left(h_{1}, h_{2}\right)=\frac{\left\langle h_{1} \mid h_{2}\right\rangle}{\sqrt{\left\langle h_{1} \mid h_{1}\right\rangle\left\langle h_{2} \mid h_{2}\right\rangle}}, \\
& \left\langle h_{1} \mid h_{2}\right\rangle=4 \Re \int_{0}^{\infty} \mathrm{d} f \frac{\tilde{h_{1}}(f) \tilde{h_{2}}(f)}{S_{h}(f)},
\end{aligned}
$$

where $S_{h}(v)\left[\mathrm{Hz}^{-1}\right]$ is the power spectral density of the strain noise from a given detector, e.g. LIGO, which were kindly provided by Shoemaker (2007, private communication) The results show good agreement with numerical deviations of a few percent, as displayed in Table 2. There, we computed the overlap of GW trains from the representative models R1E1CA $A_{L}, \mathrm{R}^{\mathrm{E}} 1 \mathrm{CA} \mathrm{A}_{L}$ and $\mathrm{R} 4 \mathrm{E} 1 \mathrm{FC} \mathrm{C}_{L}$, extracted by using Eqs. (22) and (23). We assume optimal orientation of detector and source as well as the $\mathrm{GW}$ to be emitted along the polar axis. Below, we generally apply Eq. (22) if not stated otherwise. Given our sphericallysymmetric effective GR approach to the solution of the Poisson equation, this expression is physically best motivated, since it does not depend on spatial derivatives of the gravitational potential.

We also point out that the Newtonian quadrupole formalism to extract the gravitational radiation is not gauge invariant and only valid in the Newtonian slow-motion limit. However, it was shown by Shibata \& Sekiguchi (2003) that the above method seems to be sufficiently accurate compared to more elaborate techniques, as it preserves phase while being off in amplitude by $\sim 10 \%$ in neutron star pulsations. The energy carried away by gravitational radiation can be calculated by the following expression:

$$
\begin{aligned}
E_{G W}= & \frac{c^{3}}{5 G} \int\left[\frac{\mathrm{d}}{\mathrm{d} t}\left(I_{i j}-\frac{1}{3} \delta_{i j} I_{l l}\right)\right]^{2} \mathrm{~d} t \\
= & \frac{2 c^{3}}{15 G} \int \mathrm{d} t\left[\dot{I}_{x x}^{2}+\dot{I}_{y y}^{2}+\dot{I}_{z z}^{2}\right. \\
& -\dot{I}_{x x} \dot{I}_{y y}-\dot{I}_{x x} \dot{I}_{z z}-\dot{I}_{y y} \dot{I}_{z z} \\
& \left.+3\left(\dot{I}_{x y}^{2}+\dot{I}_{x z}^{2}+\dot{I}_{y z}^{2}\right)\right]
\end{aligned}
$$

where $I_{i j}=\ddot{t}_{i j}^{T T}$. The equivalent frequency integral yields

$$
\begin{aligned}
E_{G W}= & \frac{4 c^{3}}{15 G} \int_{0}^{\infty} v^{2} \mathrm{~d} v\left[\hat{I}_{x x}^{2}+\hat{I}_{y y}^{2}+\hat{I}_{z z}^{2}\right. \\
& -\hat{I}_{x x} \hat{I}_{y y}-\hat{I}_{x x} \hat{I}_{z z}-\hat{I}_{y y} \hat{I}_{z z} \\
& \left.+3\left(\hat{I}_{x y}^{2}+\hat{I}_{x z}^{2}+\hat{I}_{y z}^{2}\right)\right],
\end{aligned}
$$

where $\hat{I}_{i j}(v)$ is the Fourier transform of the quadrupole amplitude $I_{i j}(t)$.

In order to calculate the contribution to the GW signal due to magnetic stresses, we generalised Eq. (23), taking into account contributions from the magnetic field. Following the derivations of Kotake et al. (2004a) and Obergaulinger et al. (2006), the Cartesian quadrupole gravitational wave amplitude in the MHD 
case yields

$\ddot{i}_{i j}^{T T}=\frac{G}{c^{4}} \int \mathrm{d} V\left[2 f_{i j}-\rho\left(x_{i} \partial_{j} \Phi_{\mathrm{eff}}+x_{j} \partial_{i} \Phi_{\mathrm{eff}}\right)\right]^{T T}$,

where $f_{i j}=\rho v_{i} v_{j}-b_{i} b_{j}$, and $b_{i}=B_{i} / \sqrt{4 \pi}$. Below, the GW source is assumed to be located at the Galactic centre at $R=10 \mathrm{kpc}$. We estimate the signal-to-noise ratios (SNR) for optimal filtering searches according to Flanagan \& Hughes (1998):

$$
\left(\frac{S}{N}\right)^{2}=4 \int \frac{|\hat{h}(v)|^{2}}{S_{h}(v)} \mathrm{d} v=4 \int \frac{\left|\hat{h}_{+}(v)\right|^{2}+\left|\hat{h}_{\times}(v)\right|^{2}}{S_{h}(v)} \mathrm{d} v,
$$

where $\hat{h}(v)$ is the Fourier transform of the gravitational wave amplitude, and $S_{h}(v)$ is the power spectral density of strain noise in the detector. We assume optimal orientation of detector and source. and the Fourier spectra were normalised according to Parseval's theorem.

\section{Results}

In the subsequent four subsections we will discuss the GW signature of our 25 models. While presenting the resulting GW patterns, we will pay special attention to possible imprints of different finite temperature EoS, rotation rates, nonaxisymmetric instabilities, magnetic fields and a postbounce leakage scheme on the predicted 3D GW signals. The models' initial conditions and similar relevant quantities are summarised in Table 1, whilst the GW data is listed in Table 3.

\subsection{Non- or slowly rotating core collapse}

\section{General remarks}

Non- and slowly rotating progenitors $\left(\Omega_{c, i}=0 \ldots 0.3 \mathrm{rad} \mathrm{s}^{-1}\right.$ in our model set) all undergo quasi-spherically symmetric core collapse. As the emission of GWs intrinsically depends on dynamical processes that deviate from spherical symmetry, the collapse phase in our models (that neglect inhomogenities in the progenitor star) therefore does not provide any kind of signal, as shown in Fig. 3 for $t-t_{b}<0$. However, subsequent pressure-dominated core bounce, where the collapse is halted due to the stiffening of the EoS at nuclear density $\rho_{\text {nuc }} \approx 2 \times 10^{14} \mathrm{~g} \mathrm{~cm}^{-3}$, launches a shock wave that plows through the infalling material, leaving behind a negative entropy gradient that induces so-called "prompt" convective activity (e.g. Müller et al. 2004; Buras et al. 2006a; Dimmelmeier et al. 2008; Marek et al. 2009; Ott 2009). The GW burst which is accompanied by such aspherities starts several ms after bounce when convective overturn starts to be effective. The criterion for convective instability (the "Ledoux condition") is generally expressed as (Landau \& Lifshitz 1959; Wilson \& Mayle 1988)

$\left(\frac{\partial \rho}{\partial Y_{\mathrm{e}}}\right)_{P, s}\left(\frac{\partial Y_{\mathrm{e}}}{\partial r}\right)+\left(\frac{\partial \rho}{\partial s}\right)_{P, Y_{\mathrm{e}}}\left(\frac{\partial s}{\partial r}\right)>0$,

where $Y_{\mathrm{e}}, \rho, r$ and $s$ are electron fraction, density, stellar radius and entropy per baryon respectively. Thermodynamic consistency requires $(\partial \rho / \partial s)_{Y_{\mathrm{e}}, P}<0$, which implies that a negative entropy gradient always acts in a destabilising manner. Additionally, the neutronisation burst, occurring some $\sim 5 \mathrm{~ms}$ after bounce, causes a negative lepton gradient at the edge of the PNS which further drives convection (cf. Fig. 13 of Sumiyoshi et al. 2004; Buras et al. 2006a; Dessart et al. 2006). Note that convection is be weakened in the rotational plane by positive specific angular momentum gradients in rotating cores (Endal \& Sofia 1978).
Models without deleptonisation in the postbounce phase: Effects of the EoS and magnetic fields on the GW signature

During the early postbounce stage $\left(t-t_{b} \lesssim 20-30 \mathrm{~ms}\right.$ ), prompt convective motion is predominantly driven by a negative entropy gradient, as pointed out e.g. by Scheidegger et al. (2009) (see also Dimmelmeier et al. 2008; Marek et al. 2009; Ott 2009). The GW burst to be associated with prompt convection sets in $\sim 6 \mathrm{~ms}$ after bounce in models based on the LS EoS, generally $\sim 2-3 \mathrm{~ms}$ before the same feature occurs in the corresponding simulations using the Shen EoS, as indicated in Fig. 3. The reason for this behaviour is that the shock wave in the "Shen"models carries more energy compared to those in models using a LS EoS. Therefore, the shock wave stalls at slightly later times at larger radii (see Fig. 4), and the conditions for convective activity are delayed compared to the LS runs. Note that the convective overturn causes a smoothing of the negative entropy gradient. As a result, the GW amplitude quickly decays $(t \gtrsim 30 \mathrm{~ms}$ after bounce) and is not revived during the later evolution of the models without deleptonisation in the postbounce phase, as displayed in Figs. 3 and 5. Simulations that incorporate the Shen EoS return up to a factor of 2 smaller maximum amplitudes compared to their counterparts, as can be deduced from Fig. 3 and Table 3.

We also find that the GWs from simulations which were carried out with the stiff E3 show no significant deviations from those computed with E1 (see Table 3 and Fig. 5). The minor deviations of the GWs are entirely due to the stochastic nature of convection. The waveform spectra from the LS models cover a broad frequency band ranging from $\sim 150-500 \mathrm{~Hz}$, as displayed in Fig. 6. The spectral peak of the Shen models is shifted somewhat to lower frequencies, covering a range from $\sim 150-350 \mathrm{~Hz}$ (see Fig. 6). When comparing the energy $E_{G W}$ which is emitted during the first $30 \mathrm{~ms}$ after bounce from the LS models to that of the corresponding Shen models, we find the latter models emit less energy (see Table 3). This discrepancy is due to the lower emission at higher frequency $v \gtrsim 350 \mathrm{~Hz}$ in the Shen models and the fact that the emitted energy $\mathrm{d} E_{G W} / \mathrm{d} v$ is proportional to $v^{2}$. Moreover we find that already slow rotation (rotation rate R1) leads to a deformation of the PNS. This can be quantified by considering e.g. a density cut at $\rho=10^{11} \mathrm{~g} \mathrm{~cm}^{-3}$, which is just inside the convectively unstable region. For the slowly rotating model R1E1CA, this point is located at a radial distance of $80 \mathrm{~km}$ along the polar axis at $20 \mathrm{~ms}$ after bounce. However, in the equatorial plane, the same position is reached one radial grid zone further out relative to the origin due to the action of centrifugal forces. The short time variation in the quadrupole due to rotation combines with that of prompt convection and together they lead to somewhat stronger GW emission in the slowly rotating case compared to the non-rotating model set. This effect is strongest for the GW amplitude $A_{+I I}$, which is the "axisymmetric" ( $l=2, m=0)$ component of the wave field. Despite this feature, the frequency content of models which only differ in rotation rate, stays practically unaltered.

We found the key controlling factors that govern the GW emission from prompt convection to be the i) radial location of the convectively unstable zones; and ii) the related characteristic dynamical timescales involved, for which we use as rough estimate $t_{\mathrm{dyn}} \sim \Delta_{r} / \bar{c}_{s}{ }^{1}$ (Ott 2009, private communication). Note that both i) and ii) are implicitly determined by the applied EoS, being responsible e.g. for the local speed of sound and the radial PNS density profile. Our core-collapse simulations that use a version of the LS EoS (E1 or E3) show at core bounce

\footnotetext{
$1 \overline{c_{s}}=1 / \Delta_{r} \int_{r} c_{s}(r) \mathrm{d} r$ is the radially averaged sound speed of a convectively unstable layer with a radial extension of $\Delta_{r}$.
} 
Table 3. Summary of GW related quantities ${ }^{a}$.

\begin{tabular}{|c|c|c|c|c|c|c|c|c|c|}
\hline Model & $t_{f}[\mathrm{~ms}]$ & $E_{G W}\left[M_{\odot} c^{2}\right]$ & Dir. & $\left|A_{+, b \text { max }}\right|$ & $\left|A_{\times, b, \max }\right|$ & $\left|A_{+, p b, \max }\right|$ & $\left|A_{\times, p b, \max }\right|$ & $f_{b}[\mathrm{~Hz}]$ & $f_{T W}[\mathrm{~Hz}]$ \\
\hline \multirow{2}{*}{ R0E1CA } & \multirow{2}{*}{130.8} & \multirow{2}{*}{$2.15 \times 10^{-11}$} & $\mathrm{I}$ & 3 & $<1$ & 3 & $<1$ & - & - \\
\hline & & & II & 2 & $<1$ & 5 & 1 & - & - \\
\hline \multirow{2}{*}{ R0E3CA } & \multirow{2}{*}{103.9} & \multirow{2}{*}{$5.72 \times 10^{-11}$} & I & 2 & $<1$ & 10 & $<1$ & - & - \\
\hline & & & II & 2 & $<1$ & 8 & $<1$ & - & - \\
\hline \multirow{2}{*}{ R0STCA } & \multirow{2}{*}{70.3} & \multirow{2}{*}{$1.35 \times 10^{-11}$} & I & 3 & $<1$ & 4 & $<1$ & - & - \\
\hline & & & II & 1 & $<1$ & 3 & $<1$ & - & - \\
\hline \multirow{2}{*}{ R1E1CA } & \multirow{2}{*}{112.8} & \multirow{2}{*}{$1.36 \times 10^{-10}$} & I & 3 & $<1$ & 12 & 6 & - & - \\
\hline & & & II & 3 & $<1$ & 15 & 1 & - & - \\
\hline \multirow{2}{*}{ R1E3CA } & \multirow{2}{*}{130.4} & \multirow{2}{*}{$1.43 \times 10^{-10}$} & I & 2 & $<1$ & 10 & 5 & - & - \\
\hline & & & II & 3 & $<1$ & 17 & $<1$ & - & - \\
\hline \multirow{2}{*}{ R1E1DB } & \multirow{2}{*}{112.8} & \multirow{2}{*}{$1.24 \times 10^{-10}$} & I & 3 & $<1$ & 12 & 6 & - & - \\
\hline & & & II & 3 & $<1$ & 15 & 2 & - & - \\
\hline \multirow{2}{*}{ R1STCA } & 458 & $201 \times 10^{-11}$ & I & 2 & $<1$ & 3 & 1 & - & - \\
\hline & 45.0 & $2.01 \times 10$ & II & 2 & $<1$ & 6 & $<1$ & - & - \\
\hline $\mathrm{R}^{\mathrm{E}} 1 \mathrm{CA}$ & 92.9 & $104 \times 10^{-10}$ & I & 3 & $<1$ & 4 & 2 & - & - \\
\hline NILICA & & $1.04 \times 10$ & II & 2 & $<1$ & 10 & 1 & - & - \\
\hline $\mathrm{R} 2 \mathrm{E} 1 \mathrm{AC}$ & 1273 & $5.52 \times 10^{-9}$ & I & 2 & 2 & 6 & 5 & - & - \\
\hline R $\angle \mathrm{E} I A C$ & 121.3 & $0.52 \times 10$ & II & 105 & $<1$ & 1 & 3 & 841 & - \\
\hline $\mathrm{R} 2 \mathrm{E} 3 \mathrm{AC}$ & 106.4 & $5.31 \times 10^{-9}$ & I & 2 & 2 & 4 & 4 & - & - \\
\hline & & $5.31 \times 10$ & II & 104 & $<1$ & 1 & $<1$ & 803 & - \\
\hline R2STAC & 64.0 & $7.62 \times 10^{-9}$ & I & 3 & 3 & 6 & 6 & - & - \\
\hline & 04.0 & $1.02 \times 10$ & II & 133 & $<1$ & 2 & 1 & 680 & - \\
\hline & 625 & $5.58 \times 10^{-8}$ & I & 2 & 1 & 15 & 16 & - & 725 \\
\hline R3EIAC & 02.5 & $5.58 \times 10^{0}$ & II & 393 & $<1$ & 10 & 2 & 880 & - \\
\hline R3F2 AC & 455 & $6.53 \times 10^{-8}$ & I & 2 & 1 & 11 & 13 & - & \\
\hline K $3 \mathrm{E} \angle \mathrm{AC}$ & 45.5 & $6.53 \times 10^{0}$ & II & 409 & $<1$ & 5 & 3 & 882 & - \\
\hline R3F $3 A C$ & 575 & $644 \times 10^{-8}$ & I & 2 & 2 & 6 & 9 & - & \\
\hline КJEJAC & 31.3 & $0.44 \times 10$ & II & 409 & $<1$ & 6 & 3 & 891 & - \\
\hline R3STAC & 502 & $105 \times 10^{-7}$ & I & 5 & 4 & 9 & 11 & - & 935 \\
\hline KJSIAC & 50.2 & $1.05 \times 10^{-7}$ & II & 526 & $<1$ & 5 & 3 & 854 & - \\
\hline R3E1CA & 69.4 & $8.05 \times 10^{-8}$ & I & 3 & 3 & 9 & 9 & - & - \\
\hline КJEICA & 09.4 & $8.05 \times 10^{0}$ & II & 426 & $<1$ & 5 & 3 & 897 & - \\
\hline R3E1DB & 62.7 & $776 \times 10^{-8}$ & I & 8 & 8 & 13 & 11 & - & - \\
\hline & 02.1 & $7.16 \times 10^{0}$ & II & 425 & $<1$ & 8 & 3 & 886 & - \\
\hline $\mathrm{R} 3 \mathrm{E} 1 \mathrm{AC}_{L}$ & 196.7 & $2.14 \times 10^{-7}$ & I & 1 & 1 & 136 & 136 & - & 909 \\
\hline & & $2.14 \times 10$ & II & 437 & $<1$ & 70 & 9 & 909 & - \\
\hline R4E1AC & 98.7 & $7.74 \times 10^{-8}$ & I & 1 & $<1$ & 37 & 37 & - & 662 \\
\hline & & & II & 512 & $<1$ & 20 & 6 & 385 & - \\
\hline R4STAC & 67.2 & $1.91 \times 10^{-7}$ & I & 2 & 1 & 102 & 109 & - & 902 \\
\hline & & & II & 536 & $<1$ & 53 & 3 & 396 & - \\
\hline R4E1EC & 100.8 & $7.51 \times 10^{-8}$ & I & 1 & 1 & 25 & 20 & - & 611 \\
\hline & & & II & 492 & $<1$ & 15 & 7 & 866 & \\
\hline R4E1FC & 80.1 & $7.29 \times 10^{-8}$ & I & $<1$ & $<1$ & 16 & 19 & - & 828 \\
\hline & & & 11 & $\begin{array}{r}516 \\
2\end{array}$ & $<1$ & $\begin{array}{r}9 \\
316\end{array}$ & $\begin{array}{r}3 \\
098\end{array}$ & $\begin{array}{r}859 \\
-\end{array}$ & 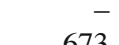 \\
\hline $\mathrm{R}_{\mathrm{EE}} 1 \mathrm{FC} \mathrm{C}_{L}$ & 97.6 & $3.42 \times 10^{-7}$ & $\begin{array}{l}1 \\
\text { II }\end{array}$ & $\begin{array}{r}2 \\
536\end{array}$ & $\begin{array}{l}1 \\
<1\end{array}$ & $\begin{array}{l}316 \\
157\end{array}$ & $\begin{array}{r}298 \\
6\end{array}$ & 485 & $\begin{array}{r}0 / 3 \\
-\end{array}$ \\
\hline & & & I & 1 & 1 & 4 & 4 & - & \\
\hline R4EICF & 19.1 & $6.50 \times 10^{-0}$ & II & 518 & $<1$ & ring-down & 2 & 370 & - \\
\hline & 032 & $120 \times 10^{-8}$ & I & 1 & 1 & 18 & 18 & - & 727 \\
\hline RJEIAC & 93.2 & $1.20 \times 10^{-0}$ & II & 238 & $<1$ & 10 & 11 & 317 & - \\
\hline
\end{tabular}

Notes. ${ }^{(a)} t_{f}$ is the time after core bounce when the simulation was stopped. $E_{G W}$ is the total energy released in gravitational radiation. We present the maximum amplitudes at different stages of their time-evolution in polar (I) and equatorial (II) direction. The subscripts $b$ and $p b$ stand for bounce and postbounce. $f_{b}$ denotes the peak frequency of the GW burst at bounce, while $f_{T W}$ stands for the spectral peak from the narrow band emission caused by a low $T /|W|$ instability. The electronic wave forms of all models are available upon request.

maximum central densities up to $\sim 25 \%$ higher than the corresponding models that apply the Shen EoS (see Table 1). Moreover, the LS-models possess a PNS which is more strongly condensed in central regions and has a steeper density gradient further out. The densities of e.g. R1E1CA and R1STCA intersect at $\sim 0.5 M_{\odot}(\sim 8 \mathrm{~km})$, as displayed in Fig. 7. In addition, models using the E1 show somewhat higher central densities compared to the ones applying the stiffer E3 variant, and densities of e.g. R0E1CA and R0E3CA cross at $\sim 7 \mathrm{~km}$. Nevertheless these two variants of the LS EoS differ only in their compressibility. The radial structures resemble each other strongly, unlike to the models carried out using the Shen EoS, as shown in Fig. 7. Hence, the particular similarity in the GW characteristics of LS runs (see Fig. 5) is not unexpected since the region which is convectively unstable is restricted to roughly the same radial- and density regimes $(R \approx 30-70 \mathrm{~km}$, 
S. Scheidegger et al.: Parameter denendent prediction of gravitational waves from stellar core collapse

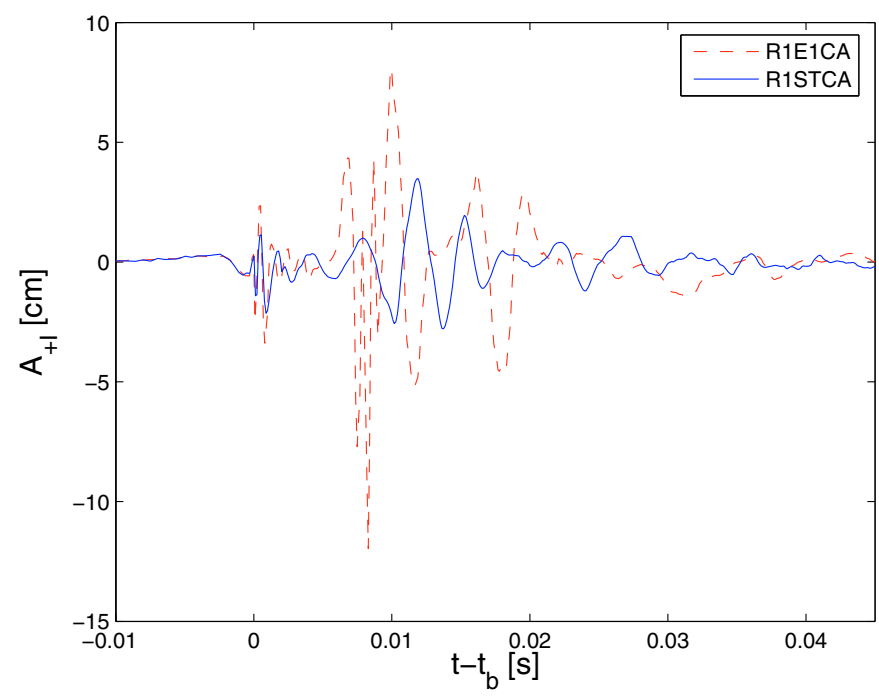

Fig. 3. Time evolution of the GW amplitude $A_{+I}$ from the slowly rotating models R1STCA (full line) and R1E1CA (dashed line). Since convection is a stochastic process, the GW amplitudes are rather insensitive to the location of the observer. Hence, we display only one representative polarisation.

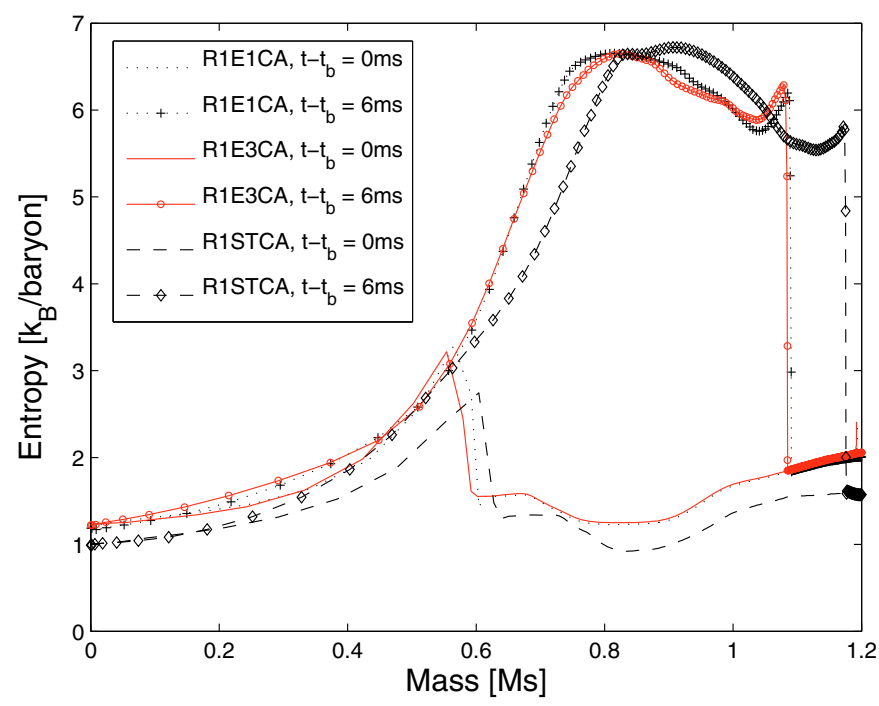

Fig. 4. Spherically averaged density profiles from the slowly rotating models R1E1CA (red full line), R1E3CA (black dotted line) and R1STCA (black dashed line) at bounce. The second entropy time slice is chosen to be approximately at the onset of the GW signal from prompt convection.

$\rho \approx 10^{11}-1.1 \times 10^{12} \mathrm{~g} \mathrm{~cm}^{-3}$ ) and therefore bound to the same dynamical timescale $t_{\text {dyn }}$ and its corresponding frequency band. For the Shen models on the other side the region of negative entropy gradient, whose boundaries are radially constrained by $R \approx 60-90 \mathrm{~km}$, contains considerably less matter than its LS analogues. This explains the smaller GW amplitudes. A narrower density spread $\left(\rho \approx 7 \times 10^{10}-1.2 \times 10^{11} \mathrm{~g} \mathrm{~cm}^{-3}\right)$ in the unstable region leads to a more restricted peak frequency band at lower values (see Fig. 4).

Computing the SNRs we find that all the simulations discussed above lie just below the detector limits of LIGO if we assume them to be located at a Galactic distance of $10 \mathrm{kpc}$. Their single-detector optimal-orientation SNR is just a little above unity. However, for a successful detection, at least a SNR of 7

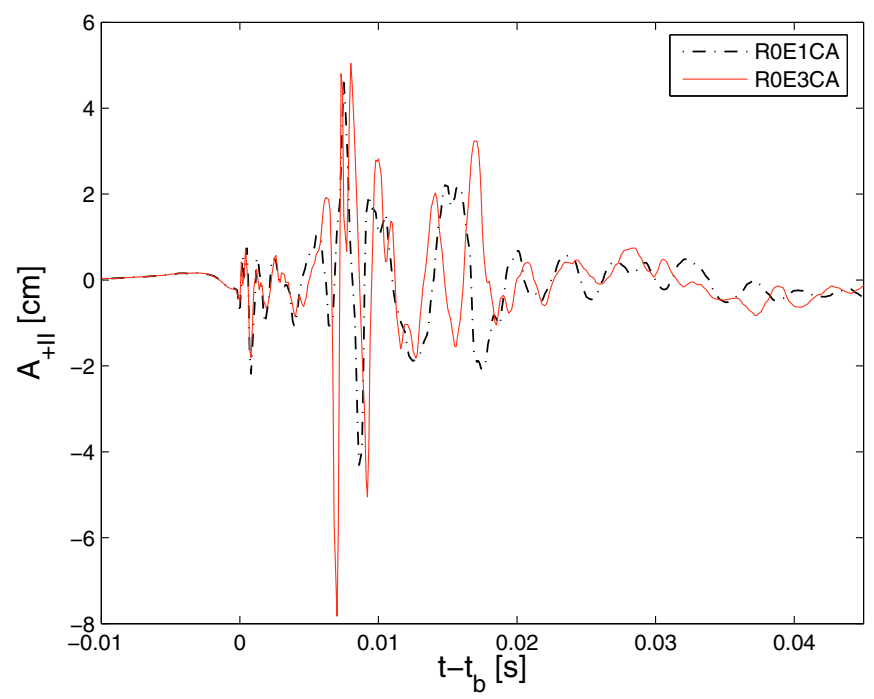

Fig. 5. Time evolution of the GW amplitude $A_{+I I}$ from the non-rotating models R0E1CA (dashed) and R0E3CA (full line).

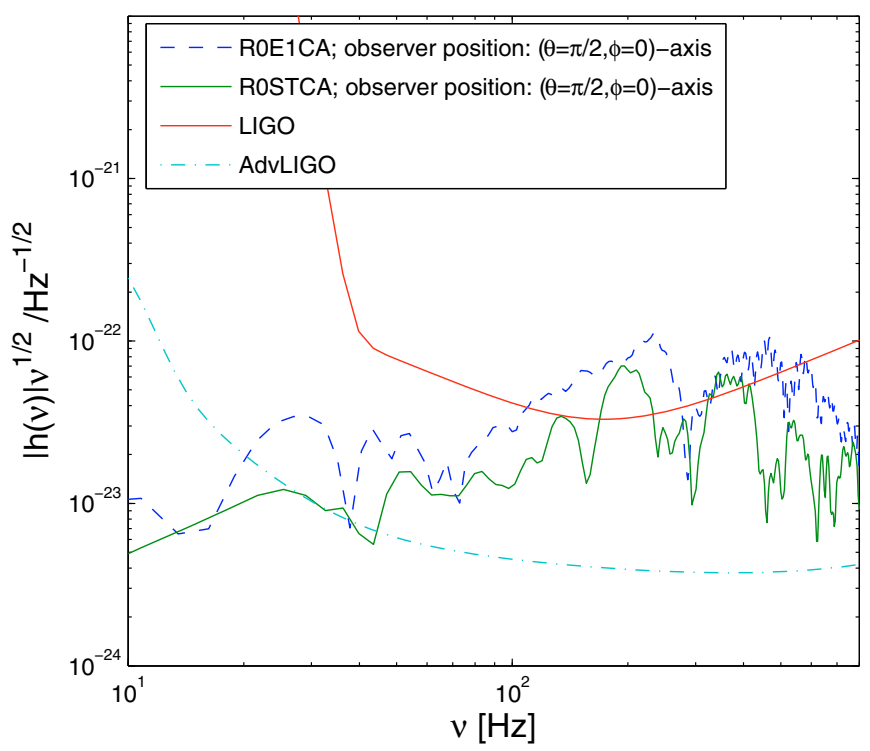

Fig. 6. Spectral energy distribution from the models R0E1CA (dashed line) and R0STCA (full line) for a spectator in the equatorial plane at a distance of $10 \mathrm{kpc}$ compared with the LIGO strain sensitivity (Shoemaker 2007, private communication) and the planned performance of Advanced LIGO. Optimal orientation between source and detector is assumed.

to 8 is necessary. Note that the SNRs of models with different EoS do not differ much at this stage since all share a similar spectral energy distribution within the window of LIGO's maximum sensitivity. The current detector sensitivity does not allow for the detection of the high frequency tail of the LS models. Note, however, that for planned future detectors such as the Advanced LIGO facility, things change dramatically. As a direct consequence, these new detectors would permit the distinction between the prompt convection GW signal from the LS and Shen EoS, since the full spectral information would be available. However, we find it impossible to discriminate between the different LS EoS variants. Hence, our simulations indicate that the GW signature depends more strongly on the asymmetry energy than the compressibility parameter of the EoS. 


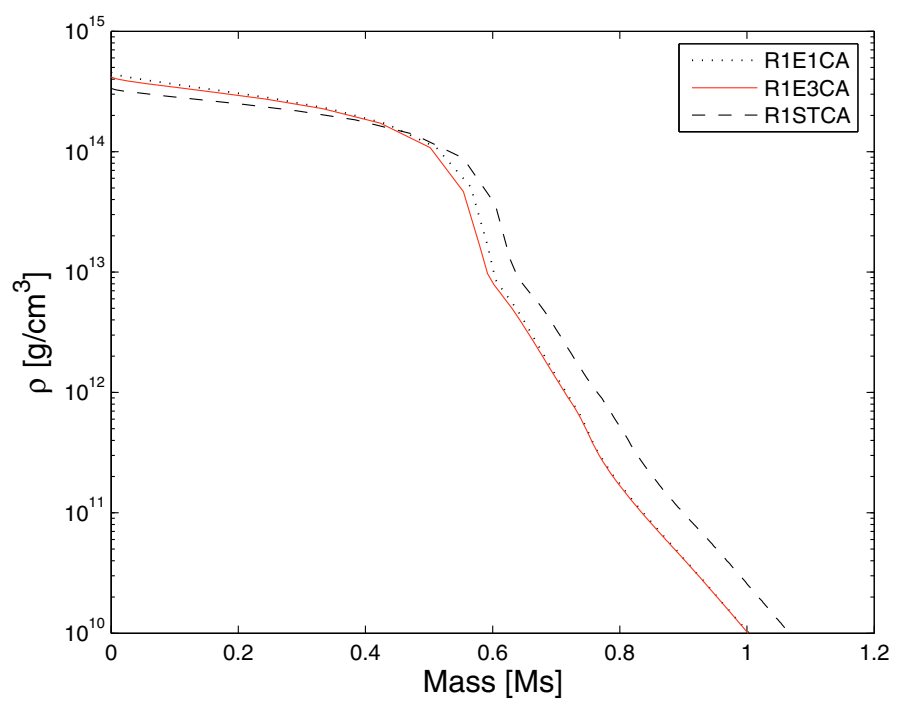

Fig. 7. Spherically averaged density profiles from models R1E1CA (dotted line), R1E3CA (full line) and R1STCA (dashed line) at core bounce $\left(t-t_{b}=0\right)$.

These results are partly different from those previously published. Recently, Marek et al. (2009) as well as Ott (2009) reported to observe GW from prompt convection in state-of-the-art 2D simulations which were launched from similar initial conditions as ours, namely the same progenitor star and the soft variant of the $\operatorname{LS} \operatorname{EoS}(K=180 \mathrm{MeV})$. Whilst the extracted GW amplitudes from early prompt convection of Marek et al. (2009) (cf. their model M15LS-2D) are in rough agreement with our results, the spectrum of their wave train peaks at considerably lower frequencies, namely around about $\sim 100 \mathrm{~Hz}$. We suppose that this discrepancy is due mainly to different radial locations of the unstable regions and the consequently encompassed amount of overturning matter. Ott (2009) computed two models with different resolution. While one of their models (s15WW95) in particular fits our results well in all characteristic GW features, namely the size of amplitudes, band of emission and the amount of emitted energy, the better resolved model (s15WW95HR) showed that convection is much weaker due to less seed perturbations and hence the GW signal and the total amount of emitted energy considerably lower. We recently also tested this issue with a better resolved model (cf. model $\mathrm{R} 1_{\mathrm{HR}}$ of Scheidegger et al. 2009, with a grid spacing of $0.6 \mathrm{~km}$ ). This better resolved model showed considerably smaller seed perturbations around $t-t_{b} \sim 0$, as grid alignment effects are better suppressed at core bounce; hence prompt convection then is much weaker and a smaller GW amplitude $(\sim 50 \%)$ is emitted. However, better numerical resolution also leads to less numerical dissipation in the system, which eases the dynamical effects that follow. Thus, Scheidegger et al. (2009) found for $\sim 10 \lesssim t \lesssim 20$ ms considerably stronger GW emission from early prompt convection compared to the $1 \mathrm{~km}$ resolved models.

Finally, we find that imposing initial magnetic fields ten times as strong as the values suggested in Heger et al. (2005) does not influence the model dynamics and therefore the GW signal at all, as can be deduced from Table 3 by a comparison of models R1E1CA and R1E1DB.

\section{Model with deleptonisation in the postbounce phase}

Due to the absence of accurate postbounce neutrino transport, it is unclear how reliably the models discussed in the previous subsection predict the GW signals from the early postbounce period. In order to investigate this question, we carried out one computationally expensive simulation, model R1E1CA ${ }_{L}$, that includes the emission of neutrinos after bounce but neglects the neutrino heating, which becomes relevant at $t-t_{b} \gtrsim 50 \mathrm{~ms}$. The absence of the latter makes it impossible for this model to track the long-term postbounce neutrino-driven convection. However, in comparison with purely MHD models, the inclusion of neutrino leakage makes is possible to distinguish effects on the GW signal due to entropy and lepton-gradient driven convection.

A detailed comparison of model R1E1CA ${ }_{L}$ with its purely magnetohydrodynamical counterpart R1E1CA shows that both follow a similar dynamical behaviour until about $20 \mathrm{~ms}$ after bounce. Asphericities leading to GW emission are predominantly driven by entropy- and not lepton-induced convection in this supernova stage. Consequently, the wave trains emitted within this early period fit each other qualitatively (cf. Figs. 3 and 8). However, we find some quantitative deviations: The GWs of R1E1CA ${ }_{L}$ reach lower maximum values (see Table 3 ), as the "Ledoux" unstable region encompasses less mass (Fig. 9), and the presence of neutrino cooling leads to a more rapid smoothing of the entropy gradient compared to the models discussed in the previous subsection. However, since the overturning matter in the top layers of the PNS has the same radial position, densities and dynamical timescales as in model R1E1CA, these models have similar GW spectra, peaking between $\sim 150-500 \mathrm{~Hz}$ (cf. Figs. 6 and 10). Therefore the physically simpler models still provide reasonably accurate $\mathrm{GW}$ predictions in frequency space until about $20 \mathrm{~ms}$ after bounce, although the amplitudes are overestimated a few $\times 10 \%$ (cf. Figs. 3 and 8). Model R1E1CA ${ }_{L}$ 's later postbounce evolution ( $t \gtrsim 20 \mathrm{~ms}$ ) differs strongly compared to its purely hydrodynamical counterpart R1E1CA. A negative radial lepton gradient, caused by the neutronisation burst and subsequent deleptonisation, drives convection inside the lower layers of the PNS (Dessart et al. 2006) at a radial position of $\sim 10-30 \mathrm{~km}$ and a density range of $\sim 10^{12}-10^{14} \mathrm{~g} \mathrm{~cm}^{-3}$ and therefore causes now the entire GW emission. The cooling PNS contracts with time, which causes the convective zones to migrate towards smaller radii and shrink. However, we point out that our leakage scheme overestimates the neutrino cooling processes, as shown in Fig. 2. Hence, this mechanism proceeds too quickly for

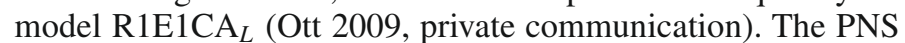
convection exhibits GW emission of roughly $\sim 0.5-1 \mathrm{~cm}$ amplitude, as can be seen in Fig. $8(t \gtrsim 20 \mathrm{~ms})$. The corresponding spectral distribution is shown in Fig. 10. A broad peak rises between $\sim 700-1200 \mathrm{~Hz}$ and reflects the dynamical timescale $t_{\text {dyn }}$ of the violent overturn activity of a millisecond scale inside the PNS. The model's SNR for LIGO at $10 \mathrm{kpc}$ is again around unity. The high frequency tail of the spectrum $(\gtrsim 700 \mathrm{~Hz})$, which is present due to PNS convection, cannot contribute to the SNR as it lies below the current detector sensitivity. Our computed GW strains for PNS convection agree roughly in amplitude with the ones found in Ott (2009) for axisymmetric MGFLD models. However, the amount of released energy emitted is found to be about one order of magnitude higher compared to his simulations. This discrepancy is most likely due to the lower average frequency content of the GWs in Ott's model $(\sim 350 \mathrm{~Hz}$, see his Fig. 7), as $\mathrm{d} E_{G W} / \mathrm{d} f \propto v^{2}$. We suppose the reason for this mismatch to be the different radial location of the convectively unstable region and thus the different related dynamical timescale $t_{\mathrm{dyn}}$. 
S. Scheidegger et al.: Parameter dependent prediction of gravitational waves from stellar core collapse
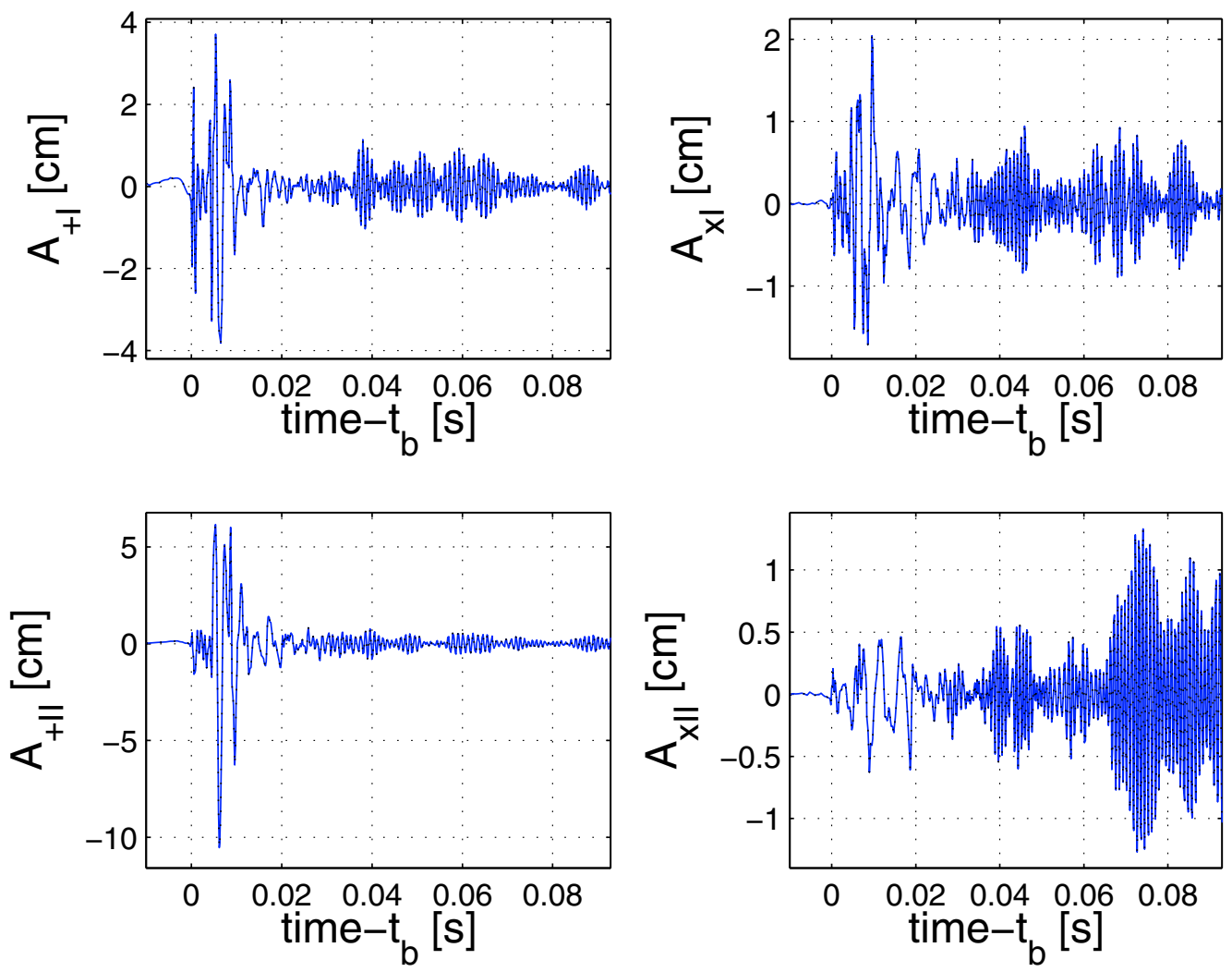

Fig. 8. Model R1E1CA ${ }_{L}$ 's time evolution of the quadrupole amplitudes $A_{+I}, A_{x I}, A_{+I I}$, and $A_{x I I}$.

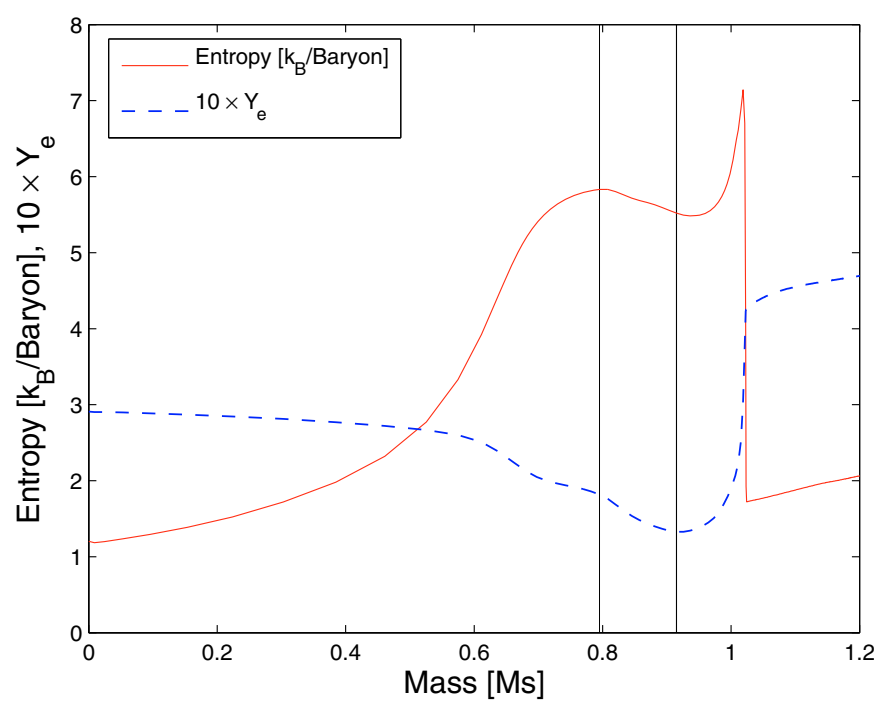

Fig. 9. Model R1E1CA ${ }_{L}$ 's spherically averaged specific entropy (full line) and $10 \times$ electron fraction $\mathrm{Y}_{\mathrm{e}}$ (dashed line) profiles are plotted versus the enclosed mass $\sim 5 \mathrm{~ms}$ after bounce. The radial position of simultaneous negativ entropy- and lepton gradients are marked by the vertical lines.

\subsection{Rapidly rotating core collapse}

\subsubsection{Core bounce}

\section{Effects of the rotation rate on the GW signature}

Rapidly rotating progenitors $\left(\Omega_{c, i}=\pi \ldots 4 \pi \mathrm{rads}^{-1}\right.$ in our model set) undergo different core-collapse dynamics compared to the previously discussed non- and slowly rotating models.

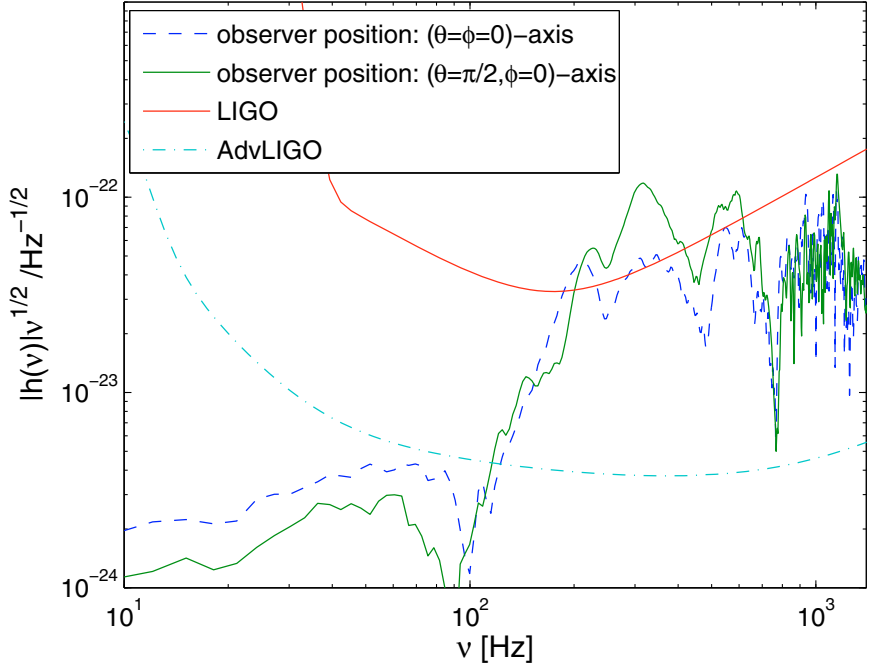

Fig. 10. Model R1E1CA ${ }_{L}$ 's spectral energy distribution of the GW signals at a distance of $10 \mathrm{kpc}$. Note that the spectrum interval from $\sim 150-500 \mathrm{~Hz}$ is caused by prompt convective activity, while the higher modes result from lepton gradient driven postbounce PNS convection.

Conservation of angular momentum in combination with contraction leads to a massive spin-up and hence oblate deformation of the core. The collapse is halted either by pure stiffening of the EoS above nuclear saturation density, or, if rotation is sufficiently strong, by a combination of the centrifugal and the nuclear forces. The abrupt slowdown of axisymmetrically axisymmetrically-arranged and quickly rotating bulk matter gives rise to rapid temporal variations in the quadrupole 

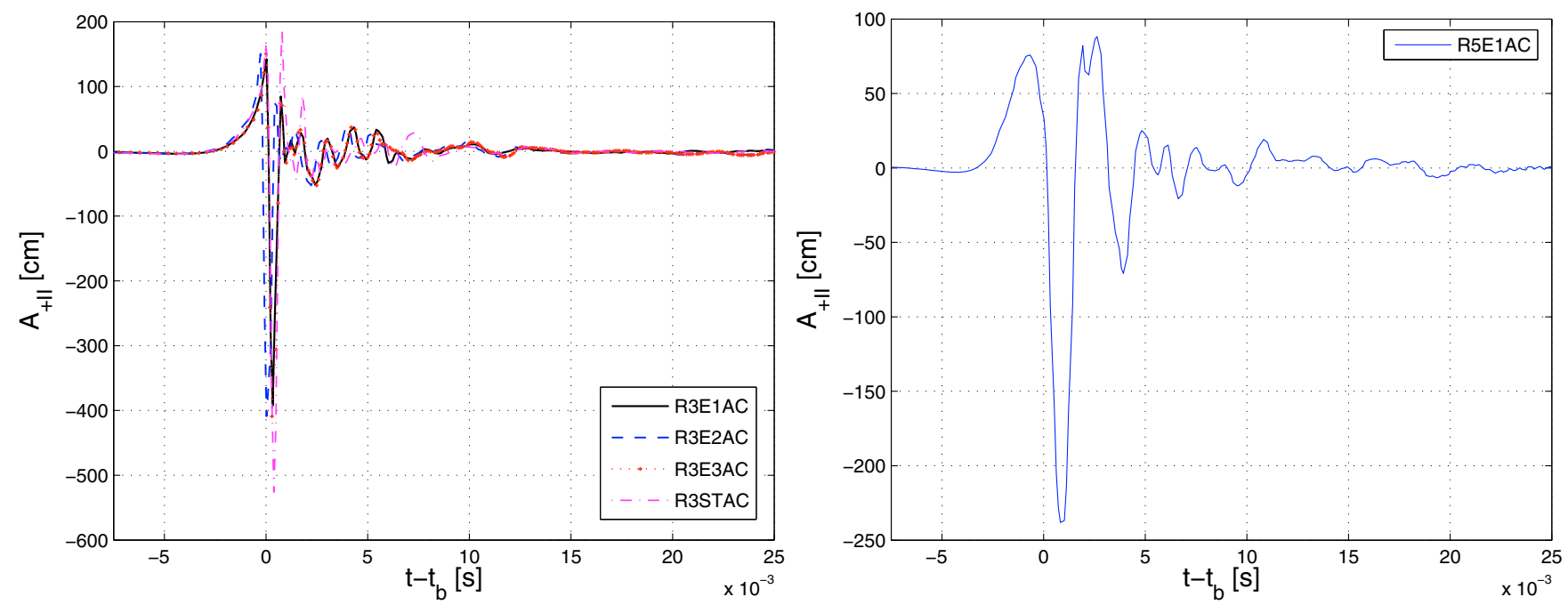

Fig. 11. Left: time evolution of the GW amplitude $\mathrm{A}_{+\mathrm{II}}$ of the models R3E1AC (solid line), R3E2AC (dashed line) R3E3AC (dotted line) and R3STAC (dashed-dotted line). Right: model R5E1AC's GW amplitude $\mathrm{A}_{+\mathrm{II}}$.

tensor, resulting in the emission of GWs. Note that the core remains essentially axisymmetric during the collapse and the early postbounce times $\left(t-t_{b} \lesssim 10 \mathrm{~ms}\right)$, as already pointed out in Ott et al. (2007a,b). Within the chosen parameter space of the rotation rate, all our models exhibit a so-called type I GW burst (Zwerger \& Müller 1997, see Fig. 11) around core bounce, no matter what the initial choice of the EoS or the magnetic field configuration. This was previously also found by Dimmelmeier et al. (2008) in 2D GR simulations without magnetic fields.

The question now arises what kind of information could possibly be delivered from a quasi-axisymmetric type I GW burst, since it is a priori unclear how degenerate it is with respect to the model parameters such as the EoS, rotation rate and so forth. This was already investigated in great detail by Dimmelmeier et al. (2008) who performed an extensive set of 2D GR corecollapse simulations. Our 3D results show the same systematics: the peak amplitude $|h|_{\max , b} \equiv \mathrm{A}_{+\mathrm{II}} / \mathrm{R}$ scales about linearly with $\beta_{b}$ for models up to a moderately rapid rotation $\left(\beta_{b} \lesssim 10 \%\right.$, see Fig. 12). The Fourier-transforms of the bounce wave trains ( $\pm 5 \mathrm{~ms}$ relative to core bounce) show for most models in the indicated parameter a spectrum with a narrow bandwidth, peaking around $\sim 800-900 \mathrm{~Hz}$ (see Table 3 ). Moreover, with growing rotation rate, prompt convective overturn in the rotational plane is suppressed by the influence of positive angular momentum gradients. This effect was first pointed out by Endal \& Sofia (1978) and is known as the Solberg-Høiland instability criterion.

The outcome of our 3D models confirms the statement of Dimmelmeier et al. (2008) that two parameters are essential for the behaviour of the $\mathrm{GW}$ amplitude around $t-t_{b}=0$, namely the mass of the inner core at bounce, which we denote as $M_{i c, b}$, and the initial central rotation rate $\Omega_{c, i}$. Over the parameter range covered by our models, $\beta_{b}$ is a strictly monotonic function of the initial central angular velocity $\Omega_{c, i}$, as displayed in Fig. 13. The mass of the inner core $M_{i c, b}$ is linked to $\beta_{b}$ via its dependence on $\Omega_{c, i}$ (see Fig. 13). The positive mass offset of the inner core (which is approximately constant for all rotational configurations) that occurs when switching from E1 to ST is interpreted as follows. In a static initial configuration, the mass of the inner core is proportional to the square of the electron fraction $Y_{\mathrm{e}}$ and the entropy per baryon (Goldreich \& Weber 1980). As the minimum of $Y_{\mathrm{e}}(\rho)$ for E1 appears at $\sim 0.276$ compared to $\sim 0.293$ for the Shen EoS (see Fig. 1), we attribute the mass

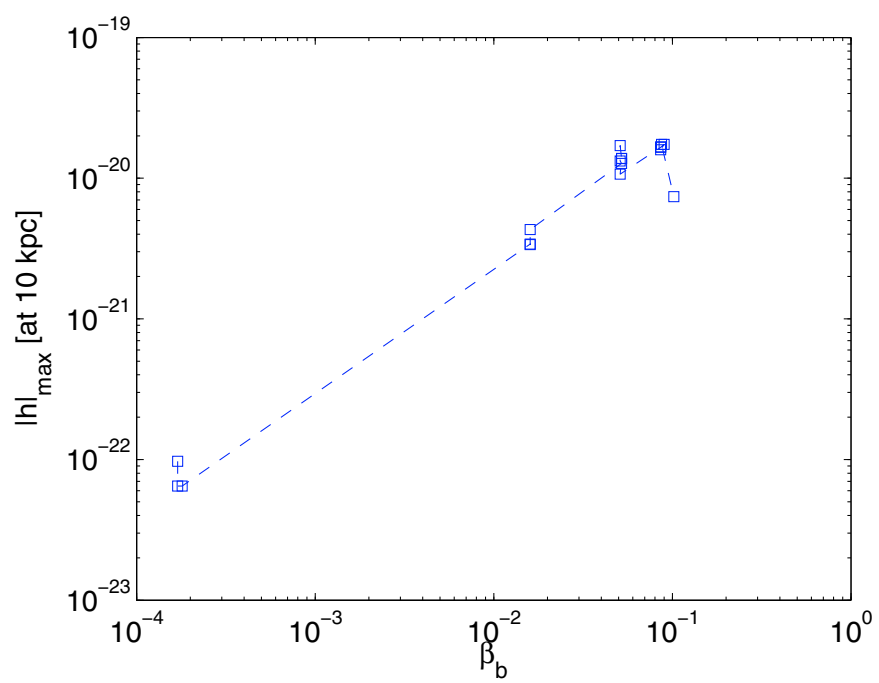

Fig. 12. Summary of all model's dimensionless peak gravitational wave amplitude $|h|_{\max , b}$ at a distance of $10 \mathrm{kpc}$ and at core bounce versus the rotation rate $\beta_{b}$. While $|h|_{\max , b}$ scales roughly linearly with $\beta_{b}$ for rotation rates $\beta_{b} \lesssim 10 \%$, the growing centrifugal force reduces $|h|_{\max , b}$ for $\beta_{b} \gtrsim 10 \%$.

difference primarily to this relative difference, and secondarily to changes in the specific entropy which occurs as the LS EoS permits more efficient electron capture. However, note that we may overestimate the spread of the inner core mass $M_{i c, b}$ in dependence with rotation compared to simulations which are carried out with full neutrino transport (Janka 2009, private communication). The stronger the rotation becomes (at $\Omega_{c, i} \gtrsim 3 \pi$ ), the increased centrifugal forces start to play a dominant role, slowing down the entire dynamics of the collapse and causing the core to rebound at sub- or just above supra-nuclear matter densities. The imprint of such behaviour is found in the GW signature by a smaller maximum amplitude and lower peak frequency compared to slower rotating models, as shown in Fig. 11 and Table 3. Similar to Dimmelmeier et al. (2008), we also find that $h_{\max }$ depends sensitively on the competition of both the amount of imposed quadrupolar deformation due to rotation, and on the other hand on the average density level in the for GW emission 


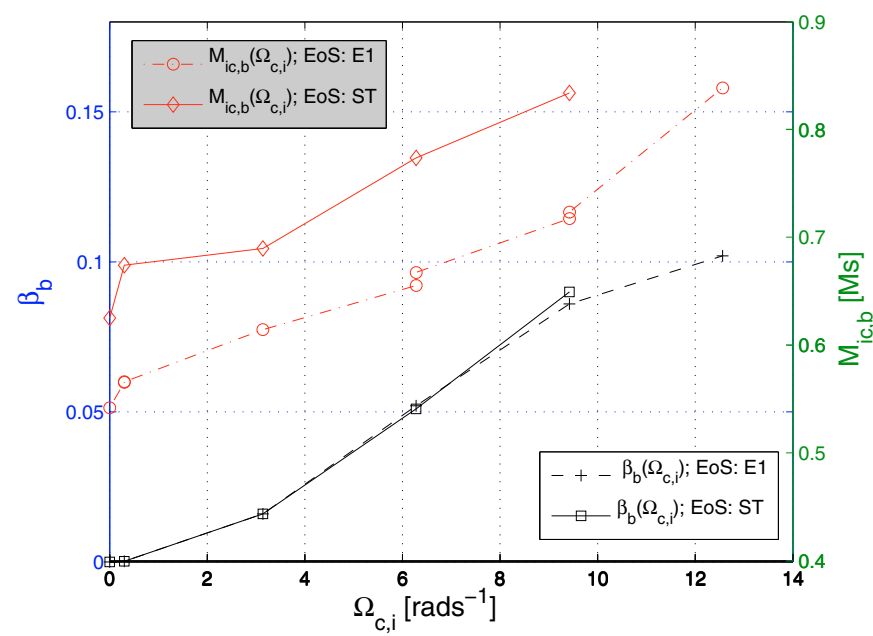

Fig. 13. Precollapse central angular velocity $\Omega_{c, i}$ versus the rotation rate rate $\beta_{b}$ or the mass of the inner core $M_{i c, b}$ at core bounce for models run with the E1 or ST EoS. We define the mass of the inner core as the mass enclosed by the entropy maximum at core-bounce, i.e. the unshocked region.

dynamically relevant region of the inner core. The density in the central region of the PNS is lowered considerably by centrifugal forces, however, no longer compensated by a prominent quadrupolar deformation which results from rapid rotation. The "optimal" configuration for strong GW emission now is overshot, which causes a smaller maximum amplitude and a lower peak frequency.

Our findings stand in very good qualitative agreement with Dimmelmeier et al. (2008), who recently performed a large set of 2D core-collapse simulations in GR, nearly identical microphysical input, but without magnetic fields. However, there are some quantitative differences. For our models that undergo a pressure-dominated bounce, we find spectra which peak in average some $100-150 \mathrm{~Hz}$ higher than the models in Dimmelmeier et al. (2008). It has been shown by Dimmelmeier (2007, private communication) that the difference stems from the fact that fully relativistic calculations shift the GW bounce spectrum to lower frequencies in comparison to the ones using an effective, spherically symmetric gravitational potential. Furthermore, for comparable precollapse rotational configurations, our models return higher peak GW values. We suspect the size of the inner core is the major cause of this difference. The mass of the inner core for all our simulations is roughly $\sim 0.1 M_{\odot}$ bigger than the ones of Dimmelmeier et al. (2008). If we take into account that we are using different electron capture rates (Bruenn 1985 versus Langanke et al. 2003; Hix et al. 2003), we consider the mismatch to be understood, as updated rates cause the mass of the inner core in our models to shrink.

\section{Effects of magnetic fields on the GW signature}

The presence of magnetic fields in our models slows down the accretion of angular momentum onto the PNS via field winding. For example, the poloidal field's stress acts on fluid particles moving in the $x-y$ plane in a direction opposing the motion, leading to a deceleration. Thus, while the GW signal from the initially "weakly" magnetised model R4E1AC is already strongly affected by centrifugal forces at bounce (the frequency peak at bounce is at $\sim 385 \mathrm{~Hz}$, while the central angular velocity is $\Omega_{c, b} \sim 6200 \mathrm{rad} \mathrm{s}^{-1}$ ), the initially more strongly magnetised but

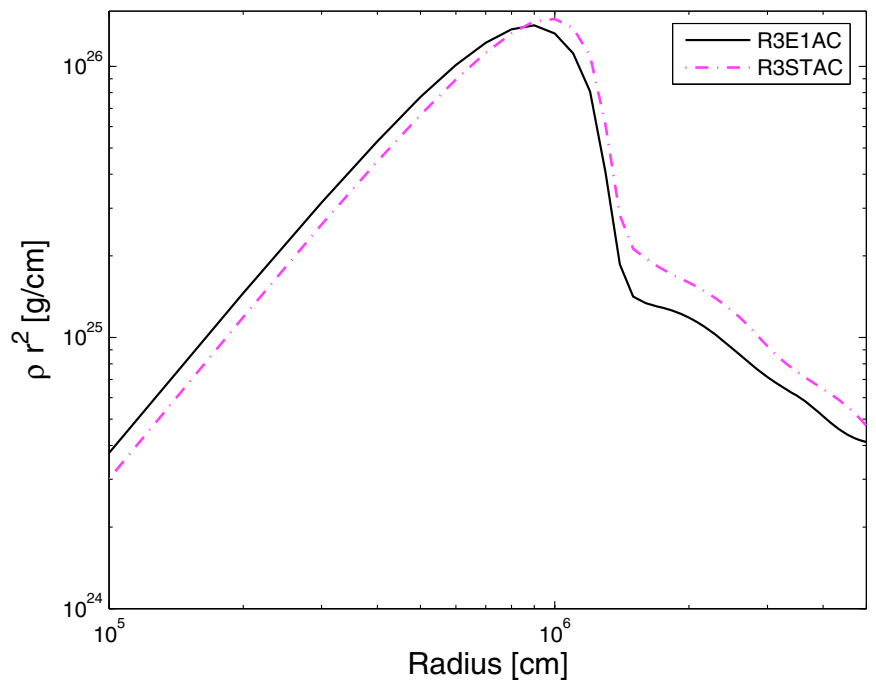

Fig. 14. Radial profiles of the weighted density $\rho r^{2}$ of the spherically averaged data at time $t=0$ relative to core bounce for model R3E1AC and its opposing model R3STAC.

otherwise comparable model R4E1FC still undergoes a pressure dominated bounce with a frequency peak at $\sim 860 \mathrm{~Hz}$ and a central angular velocity of $\Omega_{c, b} \sim 5600 \mathrm{rad} \mathrm{s}^{-1}$. However, note that this effect only gets prominent for initial magnetic fields that are by two orders of magnitude stronger than suggested by Heger et al. (2005). We will discuss the issue of strong magnetic fields in more detail in Sect. 3.2.3.

\section{Effects of the EoS on the GW signature}

In order to understand the dependence of the GW burst at bounce on the EoS, we repeated several simulations changing only the EoS while keeping the other parameters fixed. The most prominent change occurs when switching from E1 to ST. Applying the latter EoS in our models leads to systematically larger absolute GW amplitudes at lower frequencies compared to its counterparts, as shown in Table 3 and the left panel of Fig. 11. This was also observed e.g. by Kotake et al. (2004b), where the two EoS were compared. The shift to lower frequencies can be explained by the fact that the typical timescale of the GW burst at bounce is given by the free-fall timescale $\tau_{\text {dyn }} \sim 1 / \sqrt{G \bar{\rho}_{i c}}$, where $\bar{\rho}_{i c}$ is the mean density of the inner core. Since E1 leads to substantially higher central densities at core bounce compared to ST in simulations that are not dominated by centrifugal forces, the spectral peak of the GW signal is shifted to higher frequencies. The maximum GW burst amplitude depends on the dynamical timescale, the mass of the inner core as well as on the global density distribution inside the core, as pointed out e.g. in Kotake et al. (2004b) and Dimmelmeier et al. (2008). The dimensionless GW amplitude is roughly proportional to $M_{i c}$, divided by the square of the dynamical timescale $\left(h \propto M_{i c} / \tau_{\text {dyn }}^{2}\right)$. Hence, it scales approximately linearly with density. This implies that one could expect higher GW peak amplitudes from the more compact cores in the case of E1. However, since models using E1 are slightly more compact in central regions, they exhibit lower densities in the outer layers. This is displayed in Fig. 7. Following the reasoning of Dimmelmeier et al. (2008), we display the quantity $\rho r^{2}$ in Fig. 14. It is the essential quantity in the integrand of the quadrupole GW formula (see Eq. (17)). From this plot it is apparent that models run with $\mathrm{E} 1$ have higher $\rho r^{2}$ at small radii, 
ST yields higher values at intermediate and large radii. Due to their larger volume, these regions contribute more to the total quadrupole integral. For fast rotators $\left(\Omega_{c, i} \gtrsim 3 \pi\right)$, centrifugal forces start to play a dominant role, as already previously observed by Dimmelmeier et al. (2008). Here, the relative difference between the GW signatures of models run with two EoS decreases, since in the regime of lower densities, the EoS do not differ significantly. When comparing, e.g., models R3E1AC with R3STAC, the absolute size of the burst amplitudes vary roughly $25 \%$, whereas R4E1AC and R4STAC are only discriminated by $\sim 4 \%$ (see Table 3 ). In summary we state that it seems very difficult to reveal information about the different two EoS by considering the GW signature from core bounce alone. For models run with either the LS or the Shen EoS and all other parameters being indentical, the differences brought about by the EoS are clearly distinguishable. However, since a small variation in one of the other parameters can easily have a similar effect as the EoS change, it will be nearly impossible to constrain the nuclear EoS in the general case.

When changing the LS compressibility from $K=180 \mathrm{MeV}$ to $K=220 \mathrm{MeV}$ or $K=375 \mathrm{MeV}$, the features of the collapse dynamics and the corresponding GW emission remain practically unaltered, as displayed in the left panel of Fig. 11. The only notable difference occurs in the vicinity of core bounce at the center of the PNS: Models run with the softest version of the LS EoS (E1) allow the core to bounce at slightly higher central density compared to E2 and E3 (see Table 1). At the same time, models run with E1 exhibit lower densities at larger radii compared to cases where E2 or E3 was applied (see Fig. 7). However, since the differences in the radial density profiles are relatively low, these effects cancel each other if we consider again the quantity $\rho r^{2}$. This leads to GW amplitudes of similar size and frequencies.

\subsubsection{Gravitational waves from the nonaxisymmetric rotational instability}

\section{General remarks}

Rotating proto-neutron stars can be subject to non-axisymmetric rotational instabilities in situations when $T /|W|_{\text {dyn }}=\beta$ exceeds a certain critical value. Since the growing instabilities carry the object's spheroidal- into a triaxial configuration with a timedependent quadrupole moment, strong GW emission is to be expected. The best understood type of instability is the classical dynamical bar mode rotational instability with a threshold value of $\beta_{\text {dyn }} \sim 27 \%$. However, strong evidence was found by Dimmelmeier et al. (2008) that it is unlikely that the PNS reaches rotation rates required for it to become unstable during the core-collapse of and the early postbounce phase the iron core. Another possibility is the secular instability, triggered at moderately high $\beta_{\mathrm{sec}} \sim 14 \%$ if a dissipative mechanism is present. It grows on the relatively slow dissipative timescale of the order of a second (see, Tassoul 1978). Since none of our models reach such high $\beta$-values, both instabilities cannot play any role in our simulations.

However, recent work, some of which has been carried out in idealised setups and assumptions (Shibata et al. 2002; Saijo et al. 2003; Saijo \& Yoshida 2006; Watts et al. 2005; Ou \& Tohline 2006; Cerdá-Durán et al. 2007) and later also in more self-consistent core-collapse simulations (Ott et al. 2007b,a; Scheidegger et al. 2008), suggests that a differentially rotating PNS can become dynamically unstable at $T /|W|$-values as low as $\sim 1 \%$. Today this so-called low " $T /|W|$ " instability is

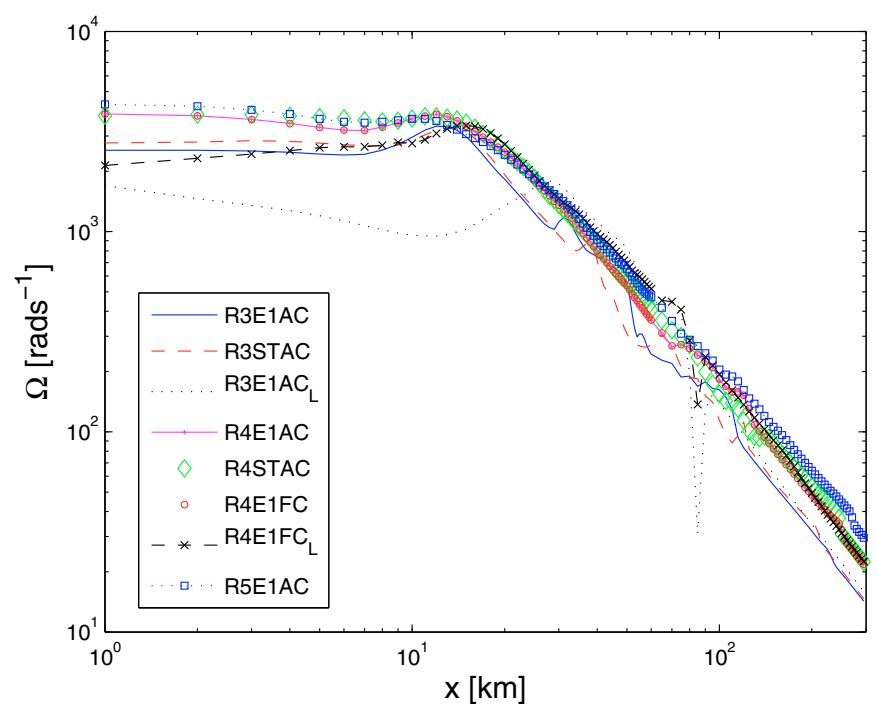

Fig. 15. Angular velocity profile along the positive $x$-axis for different models at $10 \mathrm{~ms}$ after bounce. Note that the hump in the angular velocity profile at about $\sim 11 \mathrm{~km}$ seems to be a generic feature, caused by accreting material with rather high specific angular momentum which accumulates on the nuclear density region of the PNS, as discussed in Ott et al. (2006b). It is most pronounced in models with an initial rotation rate $\lesssim \mathrm{R} 3$ and gets flatter for high initial rotation rates.

interpreted as being a resonance phenomenon (Watts et al. 2005). The underlying mechanism is suspected to be the amplification of azimuthal (non-axisymmetric) modes at co-rotation points, where the pattern speed $\sigma_{p}=\sigma / m$ of the unstable mode matches the local angular velocity, $(\propto \exp [\mathrm{i}(\sigma t-m \Phi)])$ where $\sigma$ is the mode's eigenfrequency. The PNS is differentially rotating outside a radius of $\sim 10 \mathrm{~km}$, as displayed in Fig. 15. This differential rotation provides a reservoir of shear energy may be tapped by the instability. The latter leads to spiral waves, as displayed in Fig. 16, which transfer angular momentum outwards (see, e.g., Lovelace et al. 1999). Note that this entire phenomenon appears to be closely related to the Papaloizou-Pringle instability which occurs in accretion discs around a central gravitating body (Papaloizou \& Pringle 1985).

In order to investigate the growth of the non-axisymmetric structures, we monitor the PNS by decomposing the density at a given radius $R$ in the equatorial plane $(z=0)$ into its azimuthal Fourier components:

$$
\begin{aligned}
\rho(R, z, \phi) & =\sum_{m=-\infty}^{\infty} C_{m}(R, z) \mathrm{e}^{\mathrm{i} m \phi} \\
C_{m} & =\frac{1}{2 \pi} \int_{0}^{2 \pi} \rho(R, z, \phi) \mathrm{e}^{-\mathrm{i} m \phi} \mathrm{d} \phi .
\end{aligned}
$$

The gravitational wave morphology resulting from the nonaxisymmetric process generally shows narrow-band and highly periodic signals which persist until the end of our simulations. This is shown in Fig. 17 and the upper panels of Fig. 21. Bearing in mind that the effectively measured $\mathrm{GW}$ amplitude scales with the number of GW cycles $N$ as $h_{\text {eff }} \propto h \sqrt{N}$ (see Thorne 1989) and that it could last for several hundreds of $\mathrm{ms}$ as our simulations suggest, the chances of being able to detect such kind of signal are enhanced compared e.g. to the short duration burst signal at bounce, as one can see in Fig. 18. In the lower panels of Fig. 21 the normalised mode amplitudes of the models $\mathrm{R} 3 \mathrm{E} 1 \mathrm{AC} \mathrm{C}_{L}$ and 

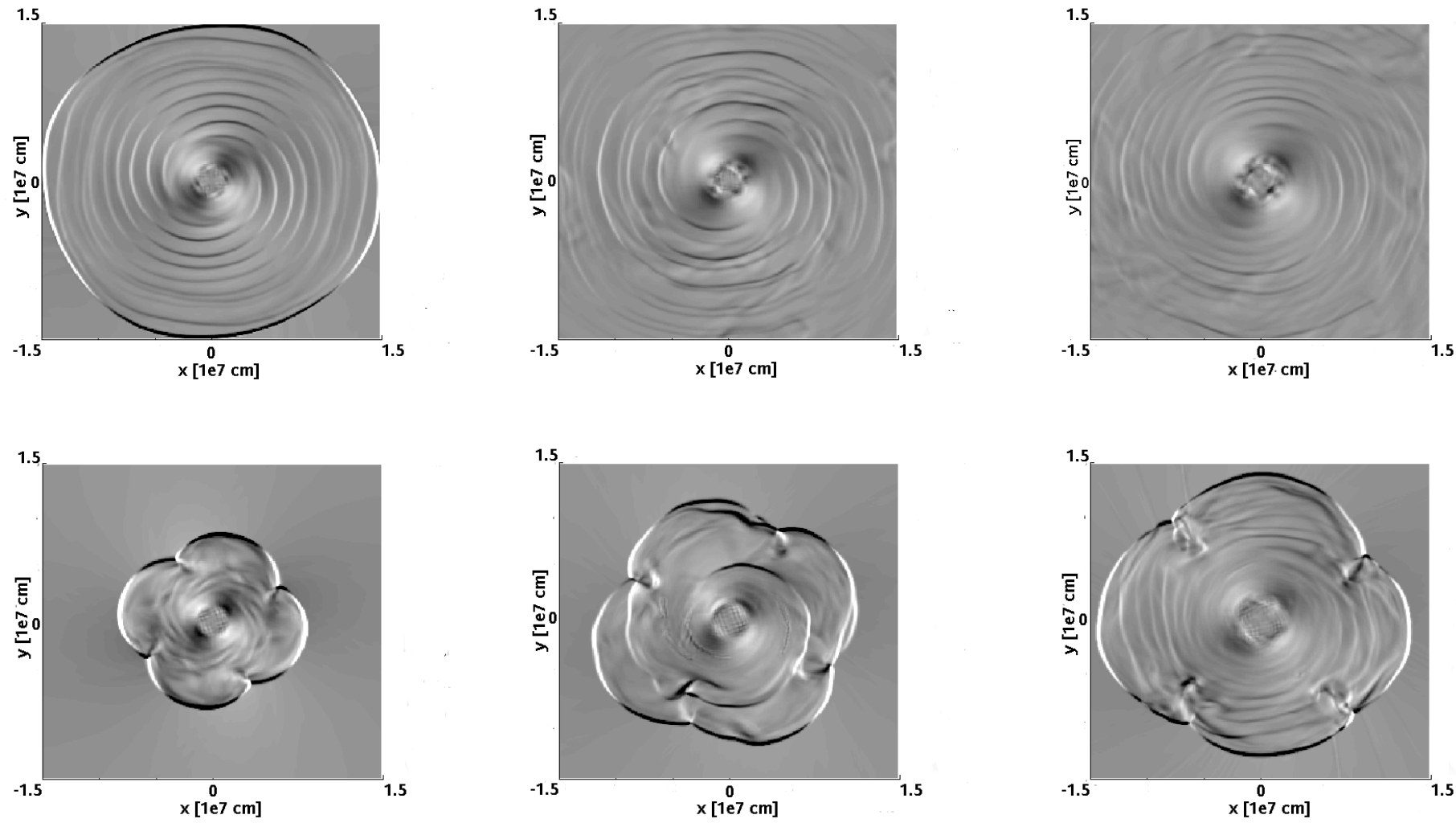

Fig. 16. Snapshots of the vorticity's $z$-component $w_{z}=(\nabla \times v)_{z}$ in the equatorial plane for models R4STAC (upper panels, $\left.t-t_{b}=10,29,63 \mathrm{~ms}\right)$ and $\mathrm{R} 4 \mathrm{E} 1 \mathrm{FC}_{L}$ (lower panels, $t-t_{b}=10,29,54 \mathrm{~ms}$ ) at three representative instants of their evolution. The innermost $300^{2} \mathrm{~km}^{2}$ are displayed, and the colour is encoded in units of $\left[\mathrm{s}^{-1}\right]$, ranging from -5000 (white) to 5000 (black). The upper panels of vorticity plots show nicely a two-armed $m=2$ spiral pattern, while the middle plot of the lower panel mainly shows the same for a $m=1$ mode.

$\mathrm{R} 4 \mathrm{E} 1 \mathrm{FC}{ }_{L}$, with

$A_{m}=\left|C_{m}\right| / C_{0}=\frac{1}{C_{0}} \sqrt{\mathfrak{R}\left(C_{m}\right)^{2}+\mathfrak{J}\left(C_{m}\right)^{2}}$,

are plotted in order to follow the behaviour of unstable modes. We generally find modes with density wave numbers $m=1,2,3$ being triggered, with the $m=1$ or $m=2$ as the overall dominant ones, depending on the individual model. Furthermore, note that all modes have the same pattern speed, as previously observed by Ott et al. (2007b,a) and Scheidegger et al. (2008). In Fig. 16, the upper panels of vorticity plots show nicely a two-armed $m=2$ spiral pattern, while the middle plot of the lower panel mainly shows the same for a $m=1$ mode, as we expect from a mode analysis of the corresponding model R4E1FC $\mathrm{F}_{L}$ presented in Fig. 21. After the early linear growth phase, the modes saturate due to Kelvin-Helmholtz shear instabilities, which break the spirals apart in the outer layers, as displayed in Fig. 16 and previously observed and discussed in e.g. Cerdá-Durán et al. (2007). Note the close relation between the $m=2$ bar mode and the emission of GWs. The growth and saturation of this mode is imprinted on the GWs emitted. As soon as it exceeds the Cartesian $m=4$ noise background, strong GW emission at a frequency corresponding to twice the $m=2$ pattern speed along the pole emerges, with the + and $\times$ polarisations shifted by a quarter cycle, as one could expect from GWs emitted by a spinning bar.

Models without deleptonisation in the postbounce phase: effects of the EoS and magnetic fields on the GW signature

We now turn the discussion in more detail to individual simulations. Models with an initial rotation rate of at least R3 $\left(\beta_{b} \sim\right.$ $5.2 \%$ ) sooner or later become low $\beta$ dynamically unstable in our parameter set (see Table 3). Note, however, that Scheidegger et al. (2009) recently found models to become dynamically unstable at even slower initial rotation rates $\left(\beta_{b} \sim 2.3 \%\right)$.

When comparing models that were carried out with the Shen- and the LS (E1) EoS, we find that the "Shen"-models emit GWs at significantly higher frequencies than their LS counterparts. This result is a consequence of the fact that the specific angular momentum, which scales as $j_{z} \propto \rho \omega_{z}$, is roughly preserved on a mass shell (Keil et al. 1996): The innermost part of the PNS in "Shen"-simulations, which rotates nearly in perfect solid body rotation at the pattern speed (see Fig. 19), has lower density in this radial region. Hence the central rotation rate must in turn be higher to fulfill the conservation law. As an example, we compare the models R3E1AC and R3STAC in detail. While R3E1AC rotates at $t-t_{b}=10 \mathrm{~ms}$ with a central rotation rate of $\omega_{\mathrm{R} 3 \mathrm{E} 1 \mathrm{AC}} \sim 2300 \mathrm{rad} \mathrm{s}^{-1}$ and has a central density $\rho_{\text {R3STAC }} \sim 3.38 \times 10^{14} \mathrm{~g} \mathrm{~cm}^{-3}$, R3STAC revolves with the values of $\omega_{\mathrm{R} 3 \mathrm{STAC}} \sim 2800 \mathrm{rad} \mathrm{s}^{-1}$ and $\rho_{\mathrm{R} 3 \mathrm{E} 1 \mathrm{AC}} \sim$ $2.63 \times 10^{14} \mathrm{~g} \mathrm{~cm}^{-3}$, as displayed in Fig. 19. Doing the maths, the ratios of $\omega_{\mathrm{R} 3 \mathrm{E} 1 \mathrm{AC}} / \omega_{\mathrm{R} 3 \mathrm{STAC}}$ to $\rho_{\mathrm{R} 3 \mathrm{STAC}} / \rho_{\mathrm{R} 3 \mathrm{STAC}}$ are about the same. We also state that the dynamical instability in the "Shen" cases grows faster generically than in the LS simulations, as shown in Fig. 17.

We generally observe slower growth of the $T /|\mathrm{W}|$ unstable modes in situations where we applied stronger initial poloidalthan toroidal magnetic fields. Although such magnetic fields ( $\left.B_{\mathrm{pol}, i}>B_{\mathrm{tor}, i}\right)$ may not be motivated by stellar evolution calculations (Heger et al. 2005), it is still important to study their effects. Model R3E1AC for example starts to emit strong GWs due to the low $T /|W|$ instability around $\sim 50 \mathrm{~ms}$ after bounce, while model R3E1DB does not within the duration of the simulation, which we followed until $\sim 65 \mathrm{~ms}$ after bounce (note, 

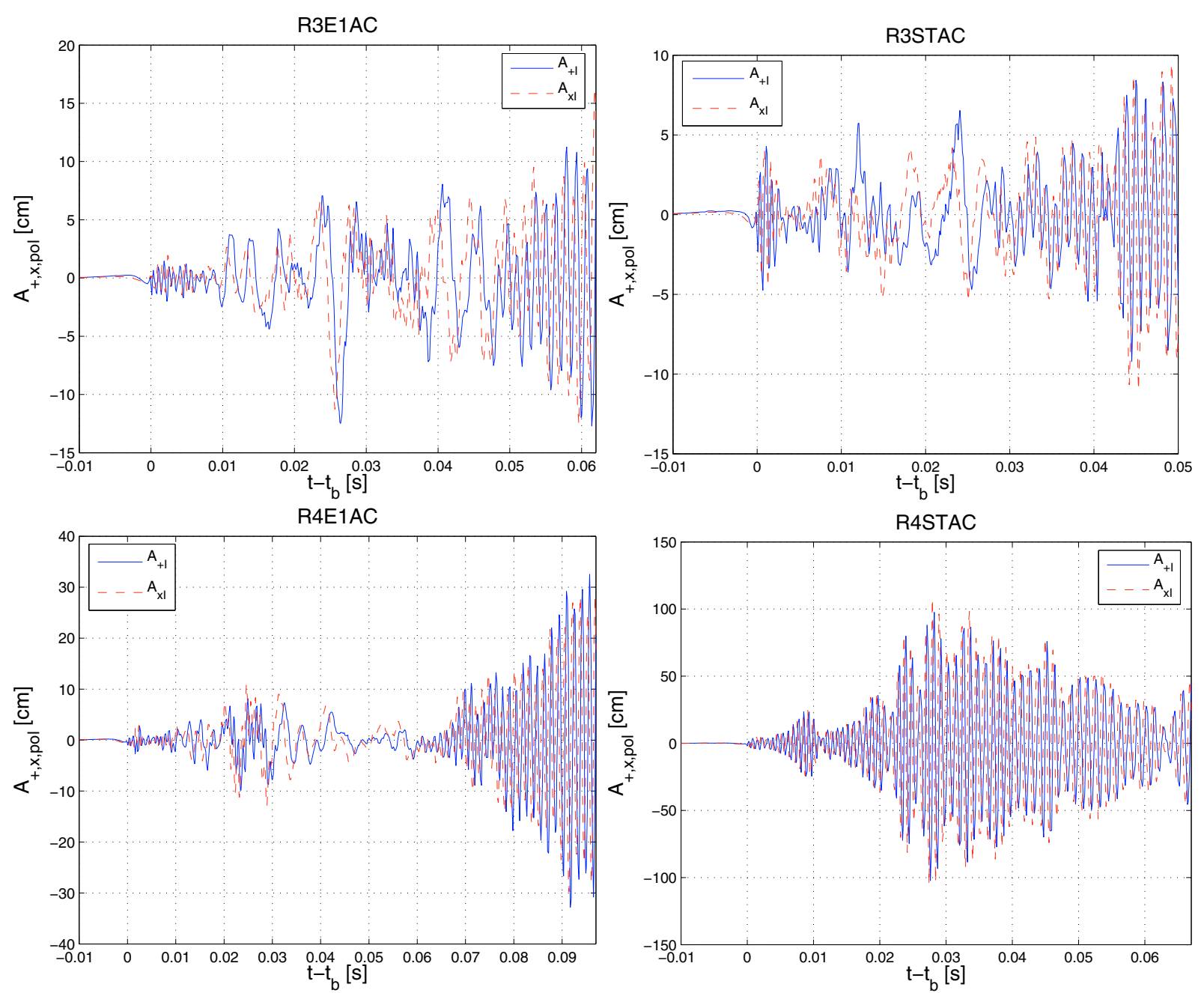

Fig. 17. The upper left panel shows the time evolution of the GW amplitudes $A_{+}$and $A_{\times}$of model R3E1AC emitted along the polar axis. The upper right displays the same for model R3STAC. The lower two panels contain the same information for R4E1AC and R4STAC from left to right. Note that $A_{+}$and $A_{\times}$oscillate at the same frequency, phase shifted by $\pi / 2$.

however, that the latter model shows strong growth of the $m=1$, 2, 3 modes although GW emission due to the low $T /|W|$ did not set in yet). The poloidal fields are able to suppress the dynamical instability for some time as they slow down the spin-up of the PNS. The detailed discussion of this issue is postponed to Sect. 3.2.3.

Centrifugal forces set a limit to the maximum frequency of the GW signal in similar fashion to the situation at core bounce. As discussed in the previous subsection, the limit is somewhere around $\sim 935 \mathrm{~Hz}$ (which is twice the pattern speed!). The faster the initial rotation rate, the stronger the influence of centrifugal forces, which slow down in the postbounce phase the advection of angular momentum onto the PNS. The result is a slower central rotation rate, a lower pattern speed and thus GW emission at lower frequencies. Beside these semi-quantitative statements which allow for the distinction of the simulations' input physics by its GW signature on a model-to-model basis, where only one parameter is varied while keeping the others fixed, it is in general very difficult to discern effects of individual features of the input physics in a GW signal that cannot unambiguously be attributed to a specific model. The degeneracy in the simulation results is large with respect to the rotation rate, the magnetic fields and the underlying EoS.

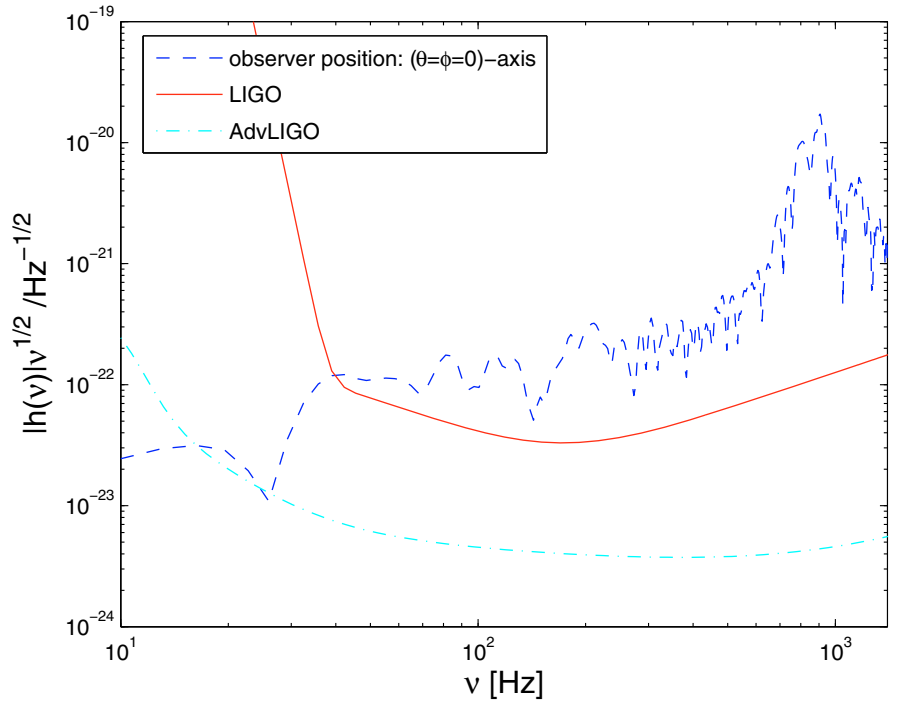

Fig. 18. Spectral energy distribution of the GW signal emitted along the polar axis from model R4STCA at a distance of $10 \mathrm{kpc}$. 

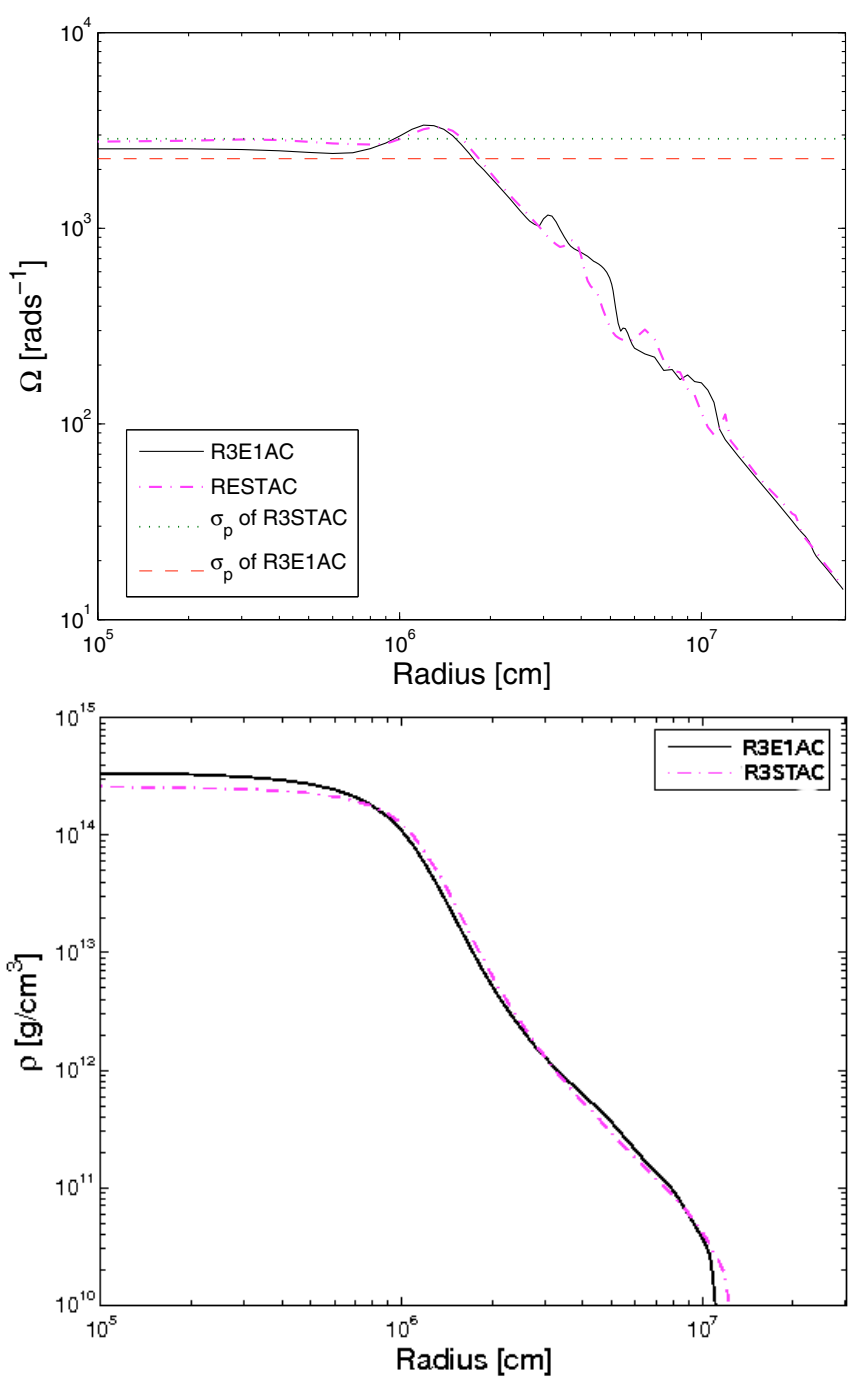

Fig. 19. The upper panel shows the angular velocity profile at $t-t_{b} \approx$ $10 \mathrm{~ms}$ along the positive $x$-axis for the models R3E1AC and R3STAC. The pattern speeds of the corresponding simulations are indicated by horizontal lines. The lower panel displays the spherically averaged radial density profiles at the same particular time.

\section{Models with deleptonisation during the postbounce phase}

The improved input physics of the two leakage models $\mathrm{R} 3 \mathrm{E} 1 \mathrm{AC}_{L}$ and $\mathrm{R} 4 \mathrm{E} 1 \mathrm{FC} C_{L}$ allows us to address the question how the inclusion of deleptonisation in the postbounce phase quantitatively alters the GW signal of our 3D MHD models. While the results show qualitative agreement with our earlier findings, they clearly deviate in quantitative terms. As the most striking feature, these models show 5-10× bigger maximum GW amplitudes due to the nonaxisymmetric dynamics compared to their counterparts that neglect neutrino cooling, as one can see in Table 3 or when comparing Fig. 17 with Fig. 21. This suggests that the treatment of postbounce neutrino cooling plays an important role when it comes to the quantitative forecast of $\mathrm{GW}$ signals from a low $\beta$ instability. The neutrino cooling during the postbounce phase leads to a more condensed PNS with a shorter dynamical timescale compared to the purely hydrodynamical treatment, as shown in Fig. 20. This in turn is directly reflected in the dynamical evolution: The shock wave stalls at considerably smaller radii and becomes more quickly unstable to azimuthal fluid modes (see Fig. 16). Since there is much more matter in the unstable region of these models, the unstable modes grow

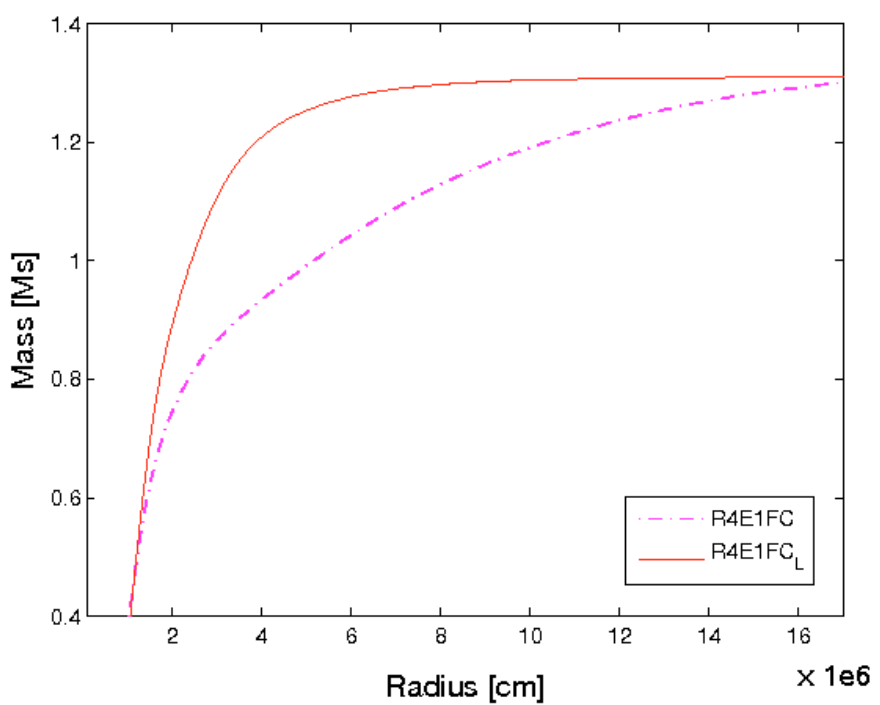

Fig. 20. The figure shows the enclosed mass $\left[M_{\odot}\right]$ as a function of radius for models $\mathrm{R} 4 \mathrm{E} 1 \mathrm{FC}$ and $\mathrm{R} 4 \mathrm{E} 1 \mathrm{FC} C_{L}$ at $t-t_{b} \approx 25 \mathrm{~ms}$, which explains the strong GW emission of the latter model.

faster and the triggered spiral density waves cause the emission of much more powerful GW.

We close this subsection by pointing out that previous corecollapse computations by Ott et al. (2007b,a) and Scheidegger et al. (2008) show both qualitative and quantitative agreement with the ones presented in the previous subsection as they incorporate nearly identical micro-physics. However, they mismatch on a quantitative scale with $\mathrm{R} 3 \mathrm{E} 1 \mathrm{AC}_{L} \& \mathrm{R} 4 \mathrm{E} 1 \mathrm{FC} C_{L}$ due to the absence of the postbounce neutrino treatment. However, we point out that our leakage scheme overestimates the compactification of the PNS due to neutrino cooling. The "reality" for the strength of GW emission therefore sould lay in between the results from the pure MHD and the leakage treatment. We also want to point out that another limitation which might affect the absolute values of the GW signature from a $T /|W|$ dynamical instability is the grid resolution. Our choice of a uniform grid leads to more than sufficient resolution at the stalled shock, but may underresolve the surface of the PNS. For example, Cerdá-Durán et al. (2007) showed that resolution can have a significant effect on the instability's developement and its GW signal.

\subsubsection{The influence of strong magnetic fields on the gravitational wave signature}

In the previous three subsections we have shown that the corecollapse dynamics and thus the GW signal of initially weakly or moderately strong magnetised stellar cores $\left(\lesssim 10^{11} \mathrm{G}\right)$ is hardly affected by magnetic fields. However, in the case of strong initial fields $\left(\sim 10^{12} \mathrm{G}\right)$ things change dramatically. The combined action of flux-freezing, field winding (Meier et al. 1976) and also of the magneto-rotational instability MRI (Balbus \& Hawley 1998) generally may lead to growth of magnetic fields by many orders of magnitude to values where the magnetic pressure reaches the order of magnitude of matter pressure. This, in turn, triggers a collimated, bipolar jet-explosion (see e.g. Burrows et al. 2007a, and references therein) by converting magnetic energy into kinetic energy. However, with our current grid setup we are unable to resolve exponential growth triggered by the MRI (Etienne et al. 2006); the initial magnetic fields are solely amplified by compression during the infall phase and by magnetic winding. 

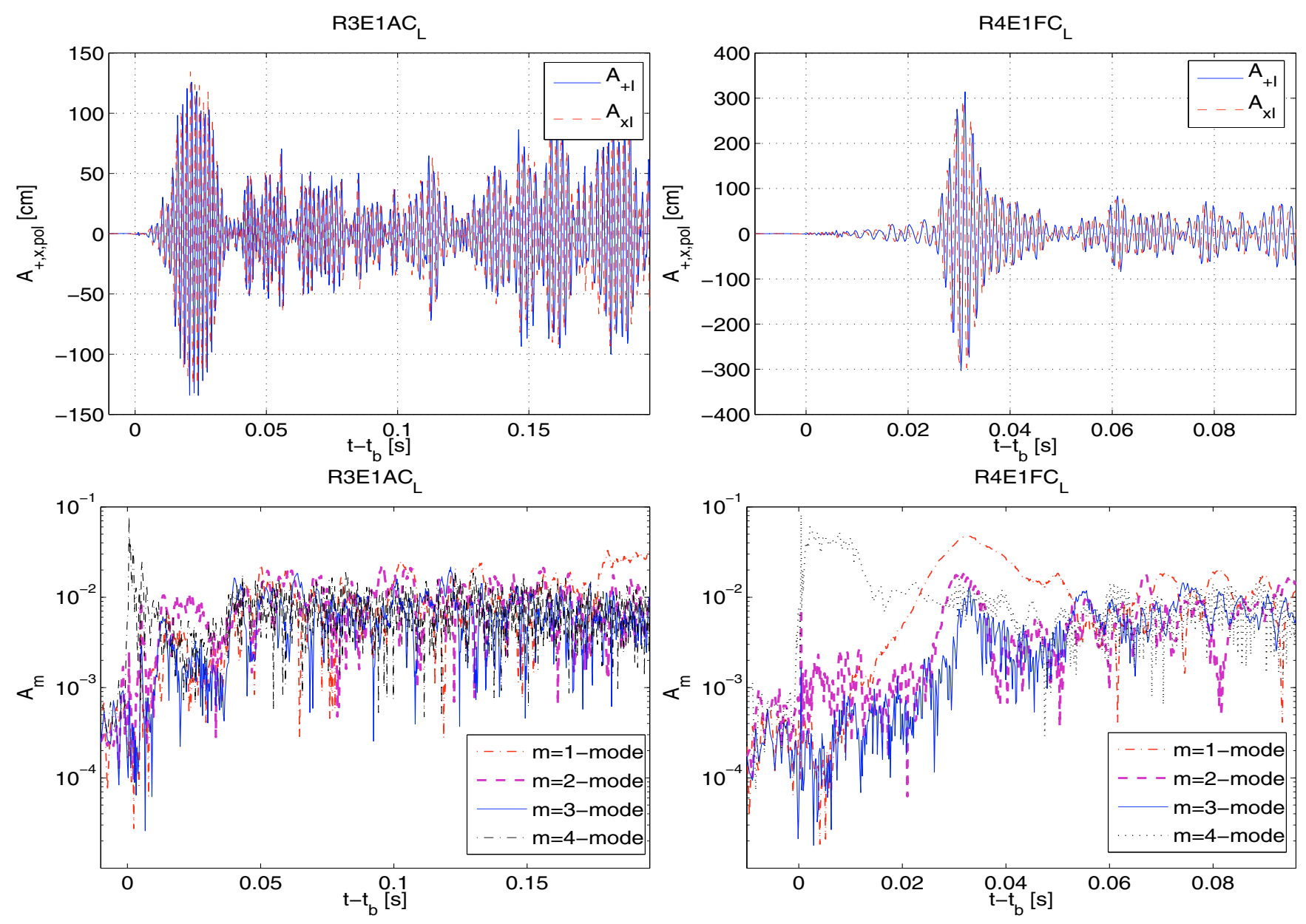

Fig. 21. The upper left panel displays the emission of the $\mathrm{A}_{+}$and the $\mathrm{A}_{\times}$-amplitude along the pole for model $\mathrm{R} 3 \mathrm{E} 1 \mathrm{AC} \mathrm{C}_{L}$. The upper right panel displays the same for model $\mathrm{R} 4 \mathrm{E} 1 \mathrm{FC}_{L}$. The lower two panels show for the same models as above the normalised mode amplitudes $A_{m}$ for $m=1,2,3,4$ extracted at a radius of $25 \mathrm{~km}$.

For two reasons, magnetically-driven explosions are of interest for the prediction of GWs. Firstly, the bipolar outflow of matter results in a "memory effect" (Thorne 1989) in the GW signature, as observed e.g. by Obergaulinger et al. (2006) and Shibata et al. (2006). As the out-stream of matter usually happens along the rotational axis, the amplitude $A_{+ \text {II }}$ will grow over time, scaling as $A_{+\mathrm{II}} \propto 2 m v_{z}^{2}$, where the ejected mass $m$ increases constantly in the early stage of a magneto-rotational supernova. Secondly, the GW amplitude of these (not necessarily) realistic models is also affected passively by strong magnetic fields, as they rise during collapse and early postbounce phase to values as high as $\sim 10^{16-17} \mathrm{G}$. The magnetic energy density may provide sizable contributions to the overall amplitude, as estimated approximately by the following formula (cf. Kotake et al. 2004b Eq. (22)):

$\eta_{\text {mag }}=\frac{\frac{B_{c}^{2}}{8 \pi}}{\rho_{c} c^{2}} \sim 10 \%\left(\frac{B_{c}}{\text { several } \times 10^{17} \mathrm{G}}\right)^{2}\left(\frac{\rho_{c}}{10^{13} \mathrm{~g} \mathrm{~cm}^{-3}}\right)^{-1}$,

where $B_{c}$ and $\rho_{c}$ stand for the central values of the magnetic field and density. If magnetic contributions become significant it is necessary to include them in Eq. (23), which then turns into Eq. (28).

For the purpose of studying the effect of very strong magnetic fields on the GW signal in 3D, we have carried out three runs: R4E1CF, R4E1FC and a leakage model R4E1FC ${ }_{L}$. In order to overcome the technically challenging simulation of magnetic field growth through small scale (Obergaulinger et al. 2009) or long-term processes, we applied initial configurations which may not be realised in nature, but can deliver the formation of jets in a similar way as natural field growth would.

Model R4E1CF, which was set up with very strong initial poloidal fields (see Table 1), shows a typical behaviour for a magneto-rotational core collapse followed by a jet-like explosion. Field compression during the collapse phase strongly amplifies both the toroidal- and poloidal magnetic fields, since fluxfreezing in stellar collapse guarantees the B-field to scale as $B \sim \rho^{2 / 3}$. Note that we evaluate toroidal and poloidal magnetic field components as:

$$
\begin{aligned}
& B_{\mathrm{tor}}=\sqrt{B_{x}^{2}+B_{y}^{2}} \\
& B_{\mathrm{pol}}=B_{z} .
\end{aligned}
$$

Furthermore, the toroidal field is build up by tapping the magnetic energy contained in the poloidal component of the field trough winding, whilst the poloidal field component grows only little during the postbounce phase by action of meridional motions in the core as shown in the lower left panel of Fig. 22. In turn, the generated hoop stresses grow fast to reach high values near the polar region, and the ratio of magnetic to matter to fluid pressure reaches $P_{\mathrm{mag}} / P_{\text {matter }} \sim 1$. A bipolar collimated jet 
S. Scheidegger et al.: Parameter dependent prediction of gravitational waves from stellar core collapse
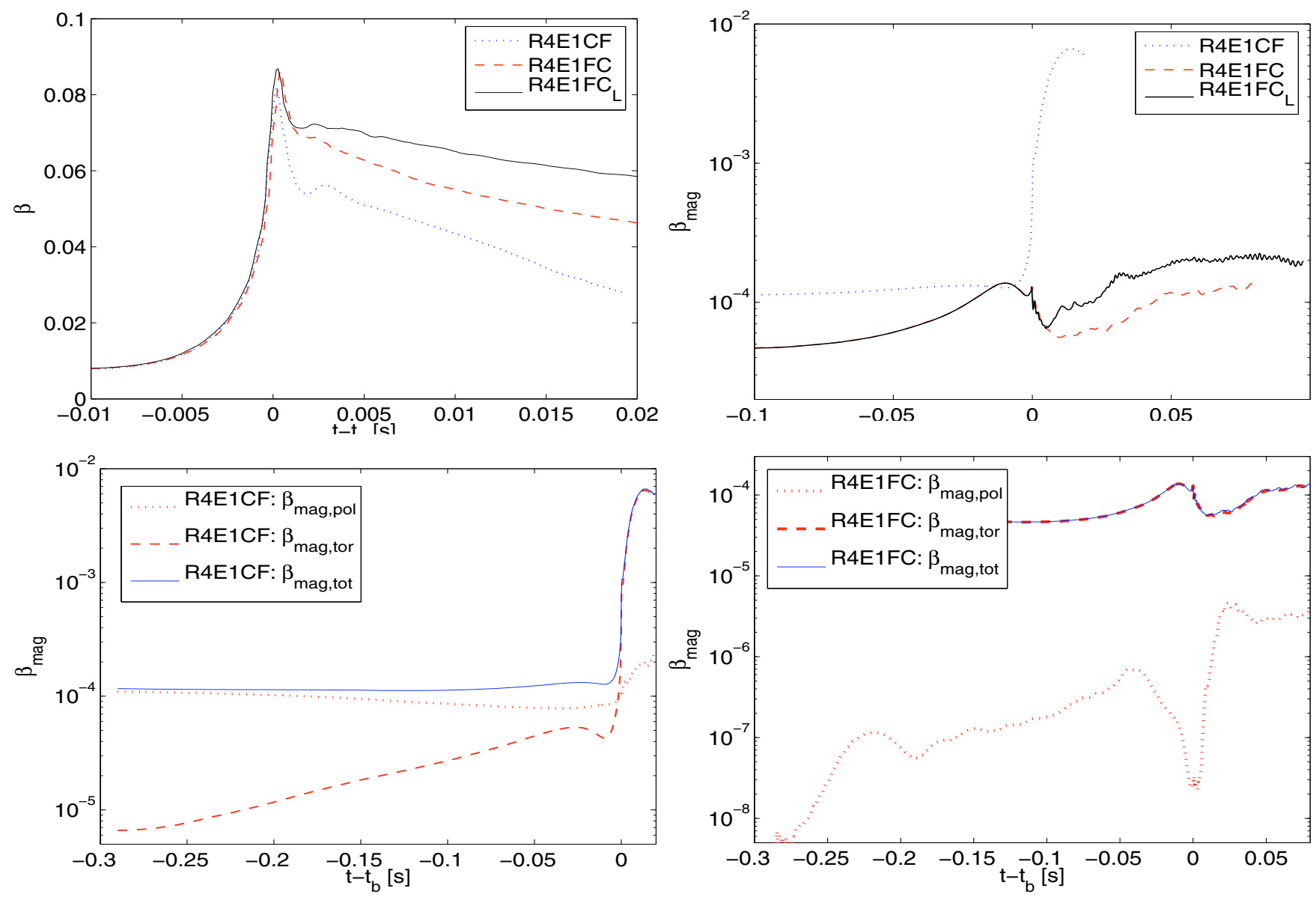

Fig. 22. The upper left panel shows the first $20 \mathrm{~ms}$ of the time evolution of the rotational energy parameter $\beta=T /|W|$ of models R4E1CF, R4E1FC and $\mathrm{R} 4 \mathrm{E} 1 \mathrm{FC}_{L}$. The upper right panel displays the time evolution of the magnetic energy parameter $\beta_{\mathrm{mag}}$ for the same models. The small hump in

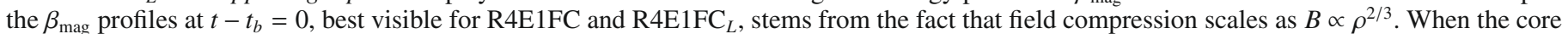
overshoots its equilibrium position at bounce and re-expands, the density decreases temporarily, which in turn is reflected in the energy density of the magnetic field. The lower left panel shows the evolution of the magnetic parameter $\beta_{\mathrm{tor}, \mathrm{mag}}, \beta_{\mathrm{pol}, \mathrm{mag}}$ and the total $\beta_{\mathrm{mag}}$ for R4E1CF, the lower right displays the same quantities for R4E1FC.

explosion is launched, quickly leaving the computational domain (see Fig. 23).

Another remarkable feature is that, in contrast to weakly magnetised models, magnetic breaking efficiently decelerates the inner core by redistributing angular momentum (Meier et al. 1976). This is shown in the upper left panel of Fig. 22. Note that model R4E1FC slows down at a higher rate than $\mathrm{R} \mathrm{E} 1 \mathrm{FC} C_{L}$ as its fluid can re-expand to larger radii due to the absence of neutrino cooling. The GW signal is displayed in Fig. 24. In order to distinguish the different contributions to the total GW amplitude $h_{i j \text {,tot }}^{T T}$, we split the magnetic and fluid part in the following way:

$h_{i j, \text { tot }}^{T T}=h_{i j, \text { matter }}^{T T}+h_{i j, \text { mag }}^{T T}$.

Around core bounce, the structure of the GW signal is very similar to that of less magnetised cores, exhibiting a clear type I signature. However, shortly after bounce we observe a growing, slowly time-varying offset of $A_{+\mathrm{II}}$ relative to the horizontal axis compared to ring-down oscillations of the weakly-magnetised simulations. There are two reasons for this behaviour. Firstly, the magnetic contribution $A_{+\mathrm{II} \text {,mag }}$ to the total GW amplitude grows strongly as magnetic forces act on the core. Secondly, as we have explained earlier in this subsection, matter-outflow along the $z$-axis also contributes to the signal. This behaviour was already observed by Obergaulinger et al. (2006) in axisymmetric simulations and its characteristical GW amplitude was named "type IV signal" (Obergaulinger et al. 2006). The contribution of the magnetic amplitudes shrinks with the onset of the jet. The emerging matter gains its kinetic energy by tapping the energy stored in the magnetic field, which causes a drop in $\beta_{\text {mag }}$ and hence also in $A_{+I I, m a g}$, as it can be seen in Figs. 22 and 24 at about $t-t_{b} \approx 10 \mathrm{~ms}$. The non-axisymmetric amplitudes are negligible compared to the axisymmetric part of the wave train (see Table 3 ) and the result of some prompt convective motions and complicated time-variations in the postbounce magnetic field configuration. However, even if a Galactic supernova was optimally orientated for the detection of a type IV signal, it is still questionable whether we could distinguish it in early stages from an ordinary type I signal. The characteristic offset of this particular signal type was suggested e.g. by Obergaulinger et al. (2006) to be a valid measure of aspherity of the ongoing supernova explosion. However, since the memory effect in the amplitude appears on the long timescale of several times $10 \mathrm{~ms}$, it would be out of the LIGO band. However, the planned spacebased DECIGO instrument (Kawamura et al. 2006) could permit to track the low frequency contribution of such a GW signal in the future. 


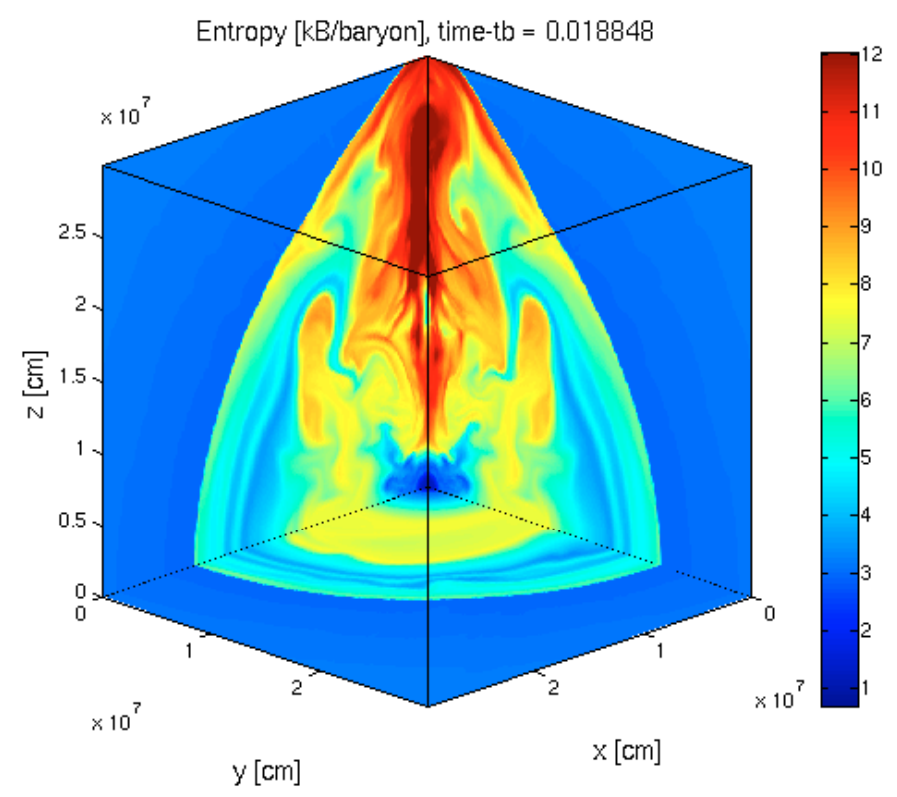

Fig. 23. Snapshot of model R4E1CF's entropy distribution in the first octant at a representative instant of its evolution. The innermost $300^{3} \mathrm{~km}^{3}$ are displayed.
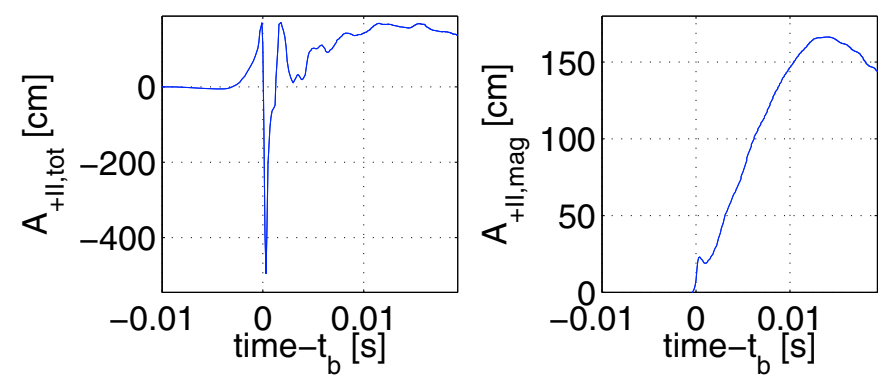

Fig. 24. Model R4E1CF's time evolution of the total quadrupole amplitude $A_{+\mathrm{II}, \text { tot }}($ left panel) and its contributions from the magnetic field $\mathrm{A}_{+\mathrm{II}, \mathrm{mag}}$ (right panel).

We point out that at the onset of jet formation around $t-t_{b} \approx$ $10 \mathrm{~ms}$, the absolute value of the magnetic field in the polar region at the edge of the PNS is $\sim 10^{16} \mathrm{G}$, which translates into a mildly relativistic fast Alfvén speed. Additionally, the velocity of the ejected matter accelerates up to radial velocities of $\sim 0.1 c$ when leaving the boundary of our computational domain. These two points challenge the Newtonian treatment of the dynamics in our scheme and suggest to use a special or general relativistic code for the further evolution of the jet dynamics.

Models R4E1FC and $\mathrm{R} 4 \mathrm{E} 1 \mathrm{FC}{ }_{L}$ show a very different dynamical outcome. For these two simulations, we assumed the initial toroidal field to be $10^{3}$ times the values of the poloidal field, as suggested from stellar evolution calculations by Heger et al. (2005). During collapse the magnetic field components primarily grow due to compression as one can see in the lower right panel of Fig. 22. Right after bounce, the strong toroidal fields in both models cause the onset of a jet, as one can see in the left panel of Fig. 25. However, surprisingly and in contrast to the previously discussed models, or simulations from Kotake et al. (2004b) where they applied similar initial conditions and obtained jet explosions in 2D, the wind-up of the poloidal- into the toroidal field does not occur efficiently enough. The spiral wave of the standing accretion shock instability (SASI, see e.g. Blondin et al. 2003), which forms at the same time, hinders and delays the growth of a jet, as displayed in the right panel of Fig. 25. We interpret this phenomenon as follows: Matter can easily slip along, but not move perpendicular to magnetic field. In the $x-y$ plane, the poloidal field's stress acts on matter in the opposite direction of the fluid motion. This is also reflected in the upper left panel of Fig. 22. It displays clearly that the magnetic forces are not capable of slowing down the inner core in case of strong initial toroidal fields as effectively as the poloidal ones that are anchored in the outer stellar layers. Since models R4E1FC and $\mathrm{R} 4 \mathrm{E} 1 \mathrm{FC}_{L}$ have relatively weak initial poloidal magnetic fields, the fluid, which rotates around the $z$-axis, can develop nearly unhindered instabilities. The developing spiral waves then counteract successfully the formation of a jet-like explosion by turning matter aside the pole (see Fig. 25), where the magnetic hoop stresses are strongest (and in axisymmetry most probably would be able to launch a jet at this stage of the simulation). This corresponds also to our observation from the last subsection where we stated that the low $T /|W|$ instability grows slower in the presence of dominant poloidal fields, because they cause stresses that act against the spiral instabilities. However, further discussion of this phenomenon is beyond the scope of this work and will be investigated in a subsequent study.

The resulting GW signals consequently show a type I signal at core bounce, subsequently followed by a low $\beta$ instability (see the right panels of 21). Although the GW contributions due to magnetic stresses in model $\mathrm{R} 4 \mathrm{E} 1 \mathrm{FC} \mathrm{F}_{L}$ show qualitatively the same features as the ones from model R4E1CF, they are smaller and dominated by the hydrodynamics part of the amplitude (e.g., $A_{+\mathrm{II}, \mathrm{mag}} \sim 10 \mathrm{~cm}$ vs. $A_{+\mathrm{II}, \text { matter }} \sim 150 \mathrm{~cm}$ at $30 \mathrm{~ms}$ after bounce).

\section{Summary and conclusions}

Core-collapse supernovae are a source of GWs that can only be modelled imperfectly due to large uncertainties in the initial conditions, input physics and the technically challenging 3D neutrino transport. The parameter space of possible initial conditions is huge since many progenitor configurations are possible at the onset of collapse. In this paper we tried to outline some dependences of the 3D GW form upon a variety of these conditions, since for un-modelled burst analysis techiques, partial information on wave forms is already useful, e.g. typical waveform features, approximate spectra and the type of polarisation and burst duration.

With our model series containing 25 three-dimensional MHD core-collapse supernova simulations, we have tried to probe the GW signature with respect to different nuclear equations of state, rotation rates, poloidal and toroidal magnetic fields, and a postbounce deleptonisation scheme.

Similar to the findings of e.g. Müller et al. (2004); Dimmelmeier et al. (2008); Marek et al. (2009); Ott (2009); Scheidegger et al. (2009), our results show that all non- and slowly rotating models release GWs due to prompt convection within the first $\sim 30 \mathrm{~ms}$ postbounce that is caused by the presence of a negative radial entropy gradient. Furthermore, we could show in simulations without deleptonisation in the postbounce phase that the waveforms obtained from this early stage of a supernova explosion contain indirect information about the underlying EoS. Due to different radial locations of the convectively unstable region and the amount of matter it contains, we were able to distinguish the LS EoS from the one of Shen. While the LS EoS leads to GW emission in a frequency band peaking between $\sim 150-500 \mathrm{~Hz}$, the spectrum of the models using the Shen EoS is restricted to roughly $\sim 150-350 \mathrm{~Hz}$. However, LIGO's current sensitivity makes it impossible to see the high-frequency 
S. Scheidegger et al.: Parameter dependent prediction of gravitational waves from stellar core collapse
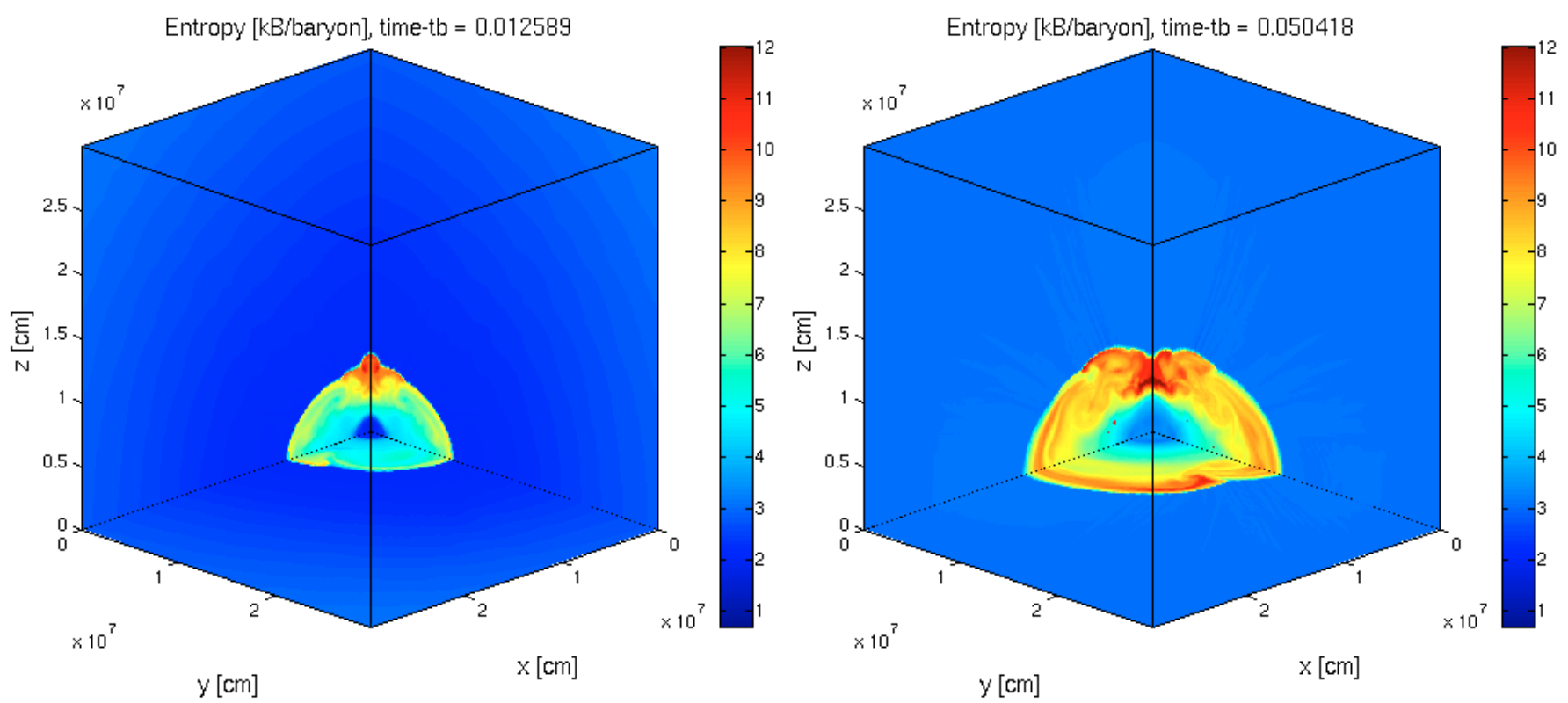

Fig. 25. Snapshots of model $\mathrm{R} 4 \mathrm{E} 1 \mathrm{FC} \mathrm{C}_{L}$ 's entropy distribution in the first octant at two representative instants of its evolution. The innermost $300^{3} \mathrm{~km}^{3}$ are displayed.

component of the LS models. Thus, a distinction between the two EoS is currently not possible. Nevertheless, planned upgrades of the interferometers in the near future should enable the discrimination between the prompt convection GW signal of the LS- and the Shen EoS. We also found minor deviations in the GW characteristics for simulations which were carried out with different compressibility versions of the LS EoS. However, the differences in the frequency domain of the GW signal are negligibly small and thus not likely to be constrained by observation.

With the inclusion of a neutrino leakage scheme in a slowly rotating model for the postbounce phase, we could show that the GWs emitted during the first $\sim 20 \mathrm{~ms}$ after bounce are predominantly due to entropy driven 'prompt' convection. In purely hydrodynamical models, the GW emission ends with the decaying negative entropy gradient. In more realistic models, a negative radial lepton gradient, caused by the neutronisation burst and the subsequent deleptonisation, takes over as driving force of the convective activity at the edge of the PNS. The long-lasting GW emission associated with this dynamical feature is roughly of the size of $\sim 1 \mathrm{~cm}$ and has a spectral peak around $\sim 700-1200 \mathrm{~Hz}$.

In our set of models, simulations with a precollapse core angular velocity within the parameter range of $\Omega_{c, i}=$ $\pi \ldots 4 \pi \mathrm{rad} \mathrm{s}^{-1}$ undergo a rotational core collapse. The models all exhibit a so-called type I GW burst at core bounce. As the most important outcome, our 3D MHD models could confirm the recent findings of Dimmelmeier et al. (2008) that the purely axisymmetric $(l=2, m=0)$ peak amplitude scales about linearly with the rotation rate $\beta_{b}$ at core bounce $\left(\left|h_{\max }\right| \propto \beta_{b}\right)$ for $\beta_{b} \lesssim 10 \%$, while the Fourier-transform of the bounce wave trains for most models in the indicated parameter range align around a spectral peak of $\sim 800-900 \mathrm{~Hz}$. However, for very fast initial rotation rates of $\Omega_{c, i} \gtrsim 3 \pi \mathrm{rad} \mathrm{s}^{-1}$, centrifugal forces significantly decelerate collapse and core bounce. The longer timescale and the weakened spin-up of the core due to the action of centrifugal forces leads generically to a decrease of the peak amplitude and a broadened spectral peak at lower frequencies. Furthermore, our results indicate that the particular choice of the nuclear EoS has little influence on the GW signal from rotational core bounce. These findings are in good qualitative agreement with the ones Dimmelmeier et al. (2008) derived from axisymmetric models.

Models with a rotation rate of $\beta_{b} \gtrsim 5 \%$ at core bounce become subject to a so-called low $T /|W|$ instability of dominant $m=1$ or $m=2$ character within the first several tens of ms after bounce, depending on the individual model. This nonaxisymmetric dynamical shear instability leads to prolonged narrow band GW emission at a frequency of twice the rotation rate of the innermost part of the PNS that rotates as a solid body. The fact that the effectively measured GW amplitude scales with the number of GW cycles $N$ as $h_{\text {eff }} \propto h \sqrt{N}$ suggests that the detection of such a signal is tremendously enhanced compared to e.g. the short-lived GW bursts from core bounce, and would allow us to probe the rotational state of the PNS over a long period. However, we point out that such a mechanism only operates if the progenitor is rotating much faster than predicted for most stars by stellar evolution calculations (Heger et al. 2005). We also find that centrifugal forces set a limit to the maximum frequency of this periodic GW signal somewhere around $\sim 935 \mathrm{~Hz}$ as they suppress the inward advection of angular momentum. Besides that, we point out that GWs from a low $T /|W|$ instability are highly degenerate with respect to initial rotation rate, EoS and magnetic fields. Thus, it is very difficult to extract individual features of the input physics from the GW signal that can clearly be attributed to the initial conditions of a progenitor. Rapidly rotating models that include the postbounce neutrino physics at a qualitative level reproduce the previous findings. However, the GW signature from these more advanced models show huge quantitative deviations from the ones that treat the postbounce phase purely hydrodynamically. As the neutrino cooling during the postbounce phase leads to a more condensed PNS, the unstable regions contain considerably more mass, which then results in 5 to 10 times bigger GW amplitudes. Motivated by these findings, we will continue to improve the postbounce neutrino physics in future simulations by including neutrino heating and the emission of $\mu / \tau$ neutrinos. 
Our simulations show that the impact of magnetic fields on the overall supernova dynamics is generally small in cores with relatively weak precollapse fields $\left(B<10^{11} \mathrm{G}\right)$. Nevertheless, if we impose very strong and probably unrealistic initial poloidal magnetic fields $\left(\sim 10^{12} \mathrm{G}\right)$, the combined action of flux-freezing and field winding allows the toroidal field component to grow over many orders of magnitude to values where the magnetic pressure can trigger a jet-like explosion along the poles. The bipolar outflow of matter then causes a type IV gravitational signal. However, if we assume a strong initial toroidal magnetic field, the onset of a jet is effectively suppressed by the fast growing spiral waves of the SASI. This finding stands in contradiction to 2D simulations, where similar configurations led to "jet"-like explosions, as e.g. in Kotake et al. (2004b). Thus, in simulations where the toroidal component of the magnetic field dominantes over the poloidal one, the magnetic contributions to the GW signal are dominated by the hydrodynamical part of the amplitude. This effect is even stronger in simulations with a deleptonisation scheme in the postbounce phase.

The major limitation of our code now is in the monopole treatment of gravity, since it cannot account for spiral structures, which could be reflected in GW. We are currently working on the improvement of this issue. The IDSA includes at present only the dominant reactions relevant to the neutrino transport problem (see Liebendörfer et al. 2009, for details). Future upgrades will also include contributions from electron-neutrino scattering, which are indispensable during the collapse phase. The inclusion of this reaction will also make the cumbersome switch of the neutrino parametrisation scheme to the IDSA at bounce obsolete. Finally, we work on the inclusion of $\mu$ and $\tau$ neutrinos, which are very important for the cooling of the PNS to its final stage as neutron star.

Acknowledgements. We thank C. D. Ott for carefully reading and commenting the manuscript. Further acknowledgements go to F.-K. Thielemann from the University of Basel for his support, John Biddiscombe and Sadaf Alam from the Swiss Supercomputing Centre CSCS for the smooth and enjoyable collaboration, and C. von Arx and E. O'Connor for helpful comments. We also thank the referee for his valuable suggestions to improve our manuscript. This work would not have been possible without the support by the Swiss National Supercomputing Centre-CSCS under project ID 168. We acknowledge support by the Swiss National Science Foundation under grant No. 200020-122287 and PP0022-106627. Moreover, this work was supported by CompStar, a Research Networking Programme of the European Science Foundation.

\section{References}

Abbott, B. et al. (LIGO Scientific) 2009, LIGO: the Laser Interferometer Gravitational-Wave Observatory, Rep. Prog. Phys., 72, 076901

Adhikari, R., Fritschel, P., \& Waldman, S. 2006, Enhanced LIGO Tech. Rep. LIGO-T060156-01-I

http://www. ligo. caltech.edu/docs/T/T060156-01.pdf

Balbus, S. A., \& Hawley, J. F. 1998, Rev. Mod. Phys., 70, 1

Blanchet, L., Damour, T., \& Schaefer, G. 1990, MNRAS, 242, 289

Blondin, J. M., Mezzacappa, A., \& DeMarino, C. 2003, ApJ, 584, 971

Bruenn, S. W. 1985, ApJS, 58, 771

Buras, R., Rampp, M., Janka, H.-T., et al. 2006a, A\&A, 447, 1049

Buras, R., Janka, H.-T., Rampp, M., et al. 2006b, A\&A, 457, 281

Burrows, A., Livne, E., Dessart, L., Ott, C. D., \& Murphy, J. 2006, ApJ, 640, 878

Burrows, A., Dessart, L., Livne, E., Ott, C. D., \& Murphy, J. 2007a, ApJ, 664, 416

Burrows, A., Livne, E., Dessart, L., Ott, C. D., \& Murphy, J. 2007b, ApJ, 655, 416

Cerdá-Durán, P., Quilis, V., \& Font, J. A. 2007, Comp. Phys. Comm., 177, 288

Dessart, L., Burrows, A., Livne, E., \& Ott, C. D. 2006, ApJ, 645, 534

Dimmelmeier, H., Font, J. A., \& Müller, E. 2002, A\&A, 393, 523

Dimmelmeier, H., Ott, C. D., Janka, H.-T., Marek, A., \& Müller, E. 2007, Phys. Rev. Lett., 98, 251101

Dimmelmeier, H., Ott, C. D., Marek, A., et al. 2008, Phys. Rev. D, 78, 064056
Endal, A. S., \& Sofia, S. 1978, ApJ, 220, 279

Eriguchi, Y., \& Müller, E. 1985, A\&A, 146, 260

Etienne, Z. B., Liu, Y. T., \& Shapiro, S. L. 2006, Phys. Rev. D, 74, 044030

Evans, C. R., \& Hawley, J. F. 1988, ApJ, 332, 659

Flanagan, É. É. \& Hughes, S. A. 1998, Phys. Rev. D, 57, 4535

Finn, L. S., \& Evans, C. R. 1990, ApJ, 351, 588

Fischer, T., Whitehouse, S. C., Mezzacappa, A., Thielemann, F.-K., \& Liebendörfer, M. 2009, A\&A, 499, 1

Fryer, C. L., \& Warren, M. S. 2004, ApJ, 601, 391

Gentile, N. A., Aufderheide, M. B., Mathews, G. J., Swesty, F. D., \& Fuller, G. M. 1993, ApJ, 414, 701

Giacomazzo, B., Rezzolla, L., \& Baiotti, L. 2009, MNRAS, 399, L164

Goldreich, P., \& Weber, S. V. 1980, ApJ, 238, 991

Heger, A., Woosley, S. E., \& Spruit, H. C. 2005, ApJ, 626, 350

Hillebrandt, W., \& Wolff, R. G. 1985, in Nucleosynthesis: Challenges and New Developments, ed. W. D. Arnett, \& J. W. Truran, 131

Hirata, K. S., Kajita, T., Koshiba, M., et al. 1988, Phys. Rev. D, 38, 448

Hix, W. R., Messer, O. E., Mezzacappa, A., et al. 2003, Phys. Rev. Lett., 91, 201102

Janka, H.-T., \& Müller, E. 1996, A\&A, 306, 167

Janka, H.-T., Langanke, K., Marek, A., Martínez-Pinedo, G., \& Müller, B. 2007, Phys. Rep., 442, 38

Kawamura, S., Nakamura, T., Ando, M., et al. 2006, Class. Quant. Grav., 23, 125 Kaeppeli, R., Whitehouse, S. C., Scheidegger, S., Pen, U.-L., \& Liebendoerfer, M. 2009 [arXiv:0910.2854]

Keil, W., Janka, H.-T., \& Müller, E. 1996, ApJ, 473, L111

Kotake, K., Yamada, S., \& Sato, K. 2003, ApJ, 595, 304

Kotake, K., Sawai, H., Yamada, S., \& Sato, K. 2004a, ApJ, 608, 391

Kotake, K., Yamada, S., Sato, K., et al. 2004b, Phys. Rev. D, 69, 124004

Kotake, K., Sato, K., \& Takahashi, K. 2006, Rep. Prog. Phys., 69, 971

Kotake, K., Ohnishi, N., \& Yamada, S. 2007, ApJ, 655, 406

Kotake, K., Iwakami, W., Ohnishi, N., et al. 2009a, ApJ, 704, 951

Kotake, K., Iwakami, W., Ohnishi, N., et al. 2009b, ApJ, 697, L133

Landau, L. D., \& Lifshitz, E. M. 1959, Fluid mechanics, Course of theoretical physics (Oxford: Pergamon Press)

Langanke, K., Martínez-Pinedo, G., Messer, O. E. B., et al. 2003, Phys. Rev. Lett., 90, 241102

Lattimer, J. M., \& Swesty, F. D. 1991, Nucl. Phys. A, 535, 331

Leblanc, J. M., \& Wilson, J. R. 1970, ApJ, 161, 541

Leonard, D. C., Filippenko, A. V., Ganeshalingam, M., et al. 2006, Nature, 440, 505

Liebendörfer, M. 2005, ApJ, 633, 1042

Liebendörfer, M., Rosswog, S., \& Thielemann, F. 2002, ApJS, 141, 229

Liebendörfer, M., Rampp, M., Janka, H.-T., \& Mezzacappa, A. 2005, ApJ, 620, 840

Liebendörfer, M., Pen, U.-L., \& Thompson, C. 2006, in International Symposium on Nuclear Astrophysics - Nuclei in the Cosmos, (NIC-IX), 132 Liebendörfer, M., Whitehouse, S. C., \& Fischer, T. 2009, ApJ, 698, 1174

Lovelace, R. V. E., Li, H., Colgate, S. A., et al. 1999, ApJ, 513, 805

Müller, E. 1982, A\&A, 114, 53

Müller, E., \& Janka, H.-T. 1997, A\&A, 317, 140

Müller, E., Rampp, M., Buras, R., Janka, H.-T., \& Shoemaker, D. H. 2004, ApJ, 603,221

Müller, B., Dimmelmeier, H., \& Müller, E. 2008, A\&A, 489, 301

Marek, A., \& Janka, H.-T. 2009, ApJ, 694, 664

Marek, A., Dimmelmeier, H., Janka, H.-T., Müller, E., \& Buras, R. 2006, A\&A, 445,273

Marek, A., Janka, H.-T., \& Müller, E. 2009, A\&A, 496, 475

Meier, D. L., Epstein, R. I., Arnett, W. D., et al. 1976, ApJ, 204, 869

Mezzacappa, A. 2005, in Supernovae as Cosmological Lighthouses, ed.

M. Turatto, S. Benetti, L. Zampieri, \& W. Shea, ASP Conf. Ser. 342, 175

Mikami, H., Sato, Y., Matsumoto, T., et al. 2008, ApJ, 683, 357

Misner, C. W., Thorne, K. S., \& Wheeler, J. A. 1973, Gravitation (San Francisco:

W.H. Freeman and Co)

Murphy, J. W., Ott, C. D., \& Burrows, A. 2009, ApJ, 707, 1173

Mönchmeyer, R., Schäfer, G., Müller, E., et al. 1991, A\&A, 246, 417

Nakazato, K., Sumiyoshi, K., \& Yamada, S. 2008, Phys. Rev. D, 77, 103006

Obergaulinger, M., Aloy, M. A., \& Müller, E. 2006, A\&A, 450, 1107

Obergaulinger, M., Cerdá-Durán, P., Müller, E., et al. 2009, A\&A, 498, 241

Ott, C. D. 2009, Class. Quant. Grav., 26, 063001

Ott, C. D., Burrows, A., Livne, E., et al. 2004, ApJ, 600, 834

Ott, C. D., Burrows, A., Dessart, L., et al. 2006a, Phys. Rev. Lett., 96, 201102

Ott, C. D., Burrows, A., Thompson, T. A., Livne, E., \& Walder, R. 2006b, ApJS, 164,130

Ott, C. D., Dimmelmeier, H., Marek, A., et al. 2007a, Phys. Rev. Lett., 98, 261101

Ott, C. D., Dimmelmeier, H., Marek, A., et al. 2007b, Class. Quant. Grav., 24, 139

Page 22 of 23 
S. Scheidegger et al.: Parameter dependent prediction of gravitational waves from stellar core collapse

Ott, C. D., Burrows, A., Dessart, L., et al. 2008, ApJ, 685, 1069

Ott, C. D., Ou, S., Tohline, J. E., et al. 2005, ApJ, 625, L119

Ou, S., \& Tohline, J. E. 2006, ApJ, 651, 1068

Papaloizou, J. C. B., \& Pringle, J. E. 1985, MNRAS, 213, 799

Pen, U.-L., Arras, P., \& Wong, S. 2003, ApJS, 149, 447

Rampp, M., Muüller, E., \& Ruffert, M. 1998, A\&A, 332, 969

Sagert, I., Fischer, T., Hempel, M., et al. 2009, Phys. Rev. Lett., 102, 081101

Saijo, M., Baumgarte, T. W., \& Shapiro, S. L. 2003, ApJ, 595, 352

Saijo, M., \& Yoshida, S. 2006, in Albert Einstein Century International Conference, AIP Conf. Ser., 861, 728

Sathyaprakash, B. S., \& Schutz, B. F. 2009, Physics, Astrophysics and Cosmology with Gravitational Waves, Living Rev. Relativity 12, 2. URL (cited on August), http://www. livingreviews. org/lrr-2009-2

Scheidegger, S., Fischer, T., Whitehouse, S. C., et al. 2008, A\&A, 490, 231

Scheidegger, S., Whitehouse, S. C., Kaeppeli, R., et al. 2009 [arXiv: 0912.1455]

Shen, H., Toki, H., Oyamatsu, K., et al. 1998a, Nucl. Phys. A, 637, 435

Shen, H., Toki, H., Oyamatsu, K., et al. 1998b, Prog. Theor. Phys., 100, 1013
Shibata, M., Karino, S., \& Eriguchi, Y. 2002, MNRAS, 334, L27

Shibata, M., Liu, Y. T., Shapiro, S. L., et al. 2006, Phys. Rev. D, 74, 104026

Shibata, M., \& Sekiguchi, Y.-I. 2003, Phys. Rev. D, 68, 104020

Sumiyoshi, K., Suzuki, H., Yamada, S., et al. 2004, Nucl. Phys. A, A730, 227

Sumiyoshi, K., Yamada, S., \& Suzuki, H. 2008, ApJ, 688, 1176

Swesty, F. D., \& Myra, E. S. 2009, ApJS, 181, 1

Takahara, M., \& Sato, K. 1988, Prog. Theor. Phys., 80, 861

Takiwaki, T., Kotake, K., \& Sato, K. 2009, ApJ, 691, 1360

Tassoul, J.-L. 1978, Theory of rotating stars, ed. J.-L. Tassoul

Thorne, K. S. 1989, in Three hundred years of gravitation, ed. S. Hawking, \& W. Israel (Cambridge University Press)

Timmes, F. X., \& Arnett, D. 1999, ApJS, 125, 277

Timmes, F. X., \& Swesty, F. D. 2000, ApJS, 126, 501

Watts, A. L., Andersson, N., \& Jones, D. I. 2005, ApJ, 618, L37

Whitcomb, S. E. 2008, Class. Quant. Grav., 25, 114013

Wilson, J. R., \& Mayle, R. W. 1988, Phys. Rep., 163, 63

Woosley, S. E. \& Weaver, T. A. 1995, ApJS, 101, 181

Zwerger, T., \& Müller, E. 1997, A\&A, 320, 209 